\title{
Extremely correlated Fermi liquid theory meets Dynamical mean-field theory: Analytical insights into the doping-driven Mott transition
}

\author{
R. Žitko, ${ }^{1,2}$ D. Hansen, ${ }^{3}$ E. Perepelitsky,${ }^{3}$ J. Mravlje, ${ }^{1}$ A. Georges,,${ }^{4,5}$ and B. S. Shastry ${ }^{3}$ \\ ${ }^{1}$ Jožef Stefan Institute, Jamova 39, SI-1000 Ljubljana, Slovenia \\ ${ }^{2}$ Faculty for Mathematics and Physics, University of Ljubljana, Jadranska 19, SI-1000 Ljubljana, Slovenia \\ ${ }^{3}$ Physics Department, University of California Santa Cruz, CA 95064, USA \\ ${ }^{4}$ Centre de Physique Théorique, École Polytechnique, CNRS, 91128 Palaiseau Cedex, France \\ ${ }^{5}$ Collège de France, 11 place Marcelin Berthelot, 75005 Paris, France \\ ${ }^{6} D P M C-M a N E P$, Université de Genève, CH-1211 Genève, Switzerland
}

(Dated: September 16, 2018)

\begin{abstract}
We consider a doped Mott insulator in the large dimensionality limit within both the recently developed Extremely Correlated Fermi Liquid (ECFL) theory and the Dynamical Mean-Field Theory (DMFT). We show that the general structure of the ECFL sheds light on the rich frequencydependence of the DMFT self-energy. Using the leading Fermi-liquid form of the two key auxiliary functions introduced in the ECFL theory, we obtain an analytical ansatz which provides a good quantitative description of the DMFT self-energy down to hole doping level $\delta \simeq 0.2$. In particular, the deviation from Fermi-liquid behavior and the corresponding particle-hole asymmetry developing at a low energy scale are well reproduced by this ansatz. The DMFT being exact at large dimensionality, our study also provides a benchmark of the ECFL in this limit. We find that the main features of the self-energy and spectral line-shape are well reproduced by the ECFL calculations in the $O\left(\lambda^{2}\right)$ 'minimal scheme', for not too low doping level $\delta \gtrsim 0.3$. The DMFT calculations reported here are performed using a state-of-the-art numerical renormalization-group impurity solver, which yields accurate results down to an unprecedentedly small doping level $\delta \lesssim 0.001$.
\end{abstract}

PACS numbers: 71.10.Ay, 71.10.Fd, 71.30.+h

\section{INTRODUCTION}

Strong electronic correlations constitute one of the major challenges in condensed-matter physics and continue to inspire new theoretical approaches. In search for novel functionalities, new materials are being synthesized on a regular basis, giving the field a steady impetus. Significant progress in the understanding of electronic correlations has been achieved from the Dynamical Mean-Field Theory (DMFT), in which the self-energy is assumed to be momentum-independent (see Ref. 1 for a review). This theory becomes exact in the limit of infinite dimensionality.

The situation in low dimensions has further challenges relating to the $\mathrm{k}$ dependence of the self energy, and thus new methods for strongly correlated electrons continue to be developed. One promising approach is Shastry's Extremely Correlated Fermi Liquid theory (ECFL), developed in a recent series of papers ${ }^{2} \mid 5$. This theory starts from the infinite- $U$ limit and is based on the Schwinger equation of motion for Gutzwiller projected electrons, these non-canonical objects requiring special attention. The theory leads to a set of analytical expressions that are in principle exact. So far, solutions of the second order expansion of these expressions in a partial projection parameter $\lambda$ are available. They can be obtained for any lattice by an iterative process analogous to the skeleton diagram method. The ECFL theory expressions have been successfully applied to account for the Angle Resolved Photo-Emission Spectroscopy (ARPES) line-shapes of cuprate superconductors $\sqrt{617}$ in the normal state.

In this work, we perform a comparative study of these two methods. We use as a test-bed the single-band doped Hubbard model at strong coupling $U$, in the limit of large dimensionality. This limit leads to simplifications in the ECFL theory which we introduce here (the details of the formalism are provided elsewhere ${ }^{8}$ ). The comparison focuses on the frequency-dependence of the self-energy and single-particle spectral line-shapes, and their evolution as the Mott insulator is approached by reducing the doping level $\delta$ (defined in Eq. (5)).

The first outcome of the present work is that, by looking at the DMFT results within an ECFL perspective, we are able to obtain new analytical insights into the DMFT description of the doping-driven Mott transition. Within the DMFT, the single-particle self-energy $\Sigma(\omega)$ displays a rich and complex frequency dependence. This has been known for some time (see e.g. Ref. 9 for a recent study), but is further investigated in the present work down to unprecedentedly low doping levels $\delta \lesssim 0.001$ using a state-of-the-art numerical renormalization group (NRG) solution of the DMFT equations. Local Fermiliquid behavior $\operatorname{Im} \Sigma \propto \omega^{2}+(\pi T)^{2}$ is obeyed only below a very low energy-scale. Above this energy scale, a marked particle-hole asymmetry develops, a feature which is beyond the Fermi liquid theory. Furthermore, the strong suppression of spectral weight in the intermediate range of energies separating the quasiparticle peak from the lower Hubbard band corresponds to a marked quasi-pole in the self-energy.

We show that all of these features can be well re- 
produced by constructing an analytical ansatz for the one-particle self-energy which is directly motivated by the ECFL construction. The ECFL introduces two key quantities, $\Psi$ and $\chi$, which play the role of auxiliary selfenergies in the Schwinger construction. The proposed analytical ansatz is obtained by retaining only the dominant Fermi-liquid terms in the low-frequency expansion of these auxiliary quantities. This is found to provide a satisfactory fit of the DMFT results for doping levels $\delta \gtrsim 0.2$. Hence, quite remarkably, the marked deviations from Fermi liquid behavior, and the particle-hole asymmetry in the physical single-particle self-energy, can be accounted for by an underlying Fermi-liquid form of the ECFL auxiliary quantities. For very large $U$, and especially for very small doping levels, additional structures appear in the DMFT results which are not present in this simplest ECFL ansatz, and presumably require additional terms beyond the Fermi liquid ones in the auxiliary functions $\Psi$ and $\chi$.

Another synergistic outcome of our study is that, because the DMFT provides an exact solution in the limit of large dimensionality, it can be used to benchmark the ECFL in this limit. We present here quantitative results obtained within the 'minimal scheme' implementation of the ECFL in high dimensions ${ }^{8}$, giving rise to an expansion to order $\lambda^{2}$ in the projection parameter $\lambda$. We find that the main features of the self-energy and the spectral line-shape are well reproduced by the $O\left(\lambda^{2}\right)$ ECFL calculations, on a semi-quantitative level, for not too low doping $\delta \gtrsim 0.3$. Improvement will require further developments of the ECFL approach. Since the DMFT is able to handle any finite $U$, while the ECFL construction is motivated by the very large $U$ limit, this comparison also sheds light on the adiabatic connection between the regime of moderate and extreme correlations.

We emphasize that ECFL can be used on two different levels. On one level, it provides a functional form for the physical Green's function and the corresponding selfenergy in terms of the auxiliary ECFL self-energies $\Psi(\omega)$ and $\chi(\omega)$. By assuming the simplest Fermi-liquid form for these two self-energies over a certain frequency range centered around $\omega=0$, we successfully fit the physical self-energy obtained through DMFT in this frequency range for $\delta \gtrsim 0.2$. This is remarkable since the frequency range used is substantially larger than the characteristic frequency at which the physical self-energy begins to deviate from Fermi-liquid behavior, and even encompasses the quasi-pole in the physical self-energy at negative frequencies. This phenomenological approach to ECFL is the one used in the first five sections of the paper, and the results of this fit are displayed in Figs. 8, 9, and 10. On the second level, ECFL provides a microscopic theory by which one can obtain concrete results for $\Psi$ and $\chi$ via an expansion in the projection parameter $\lambda$. In the remainder of the paper, the results obtained from the $O\left(\lambda^{2}\right)$ theory are benchmarked against the results obtained from DMFT, which are exact in the limit of infinite dimensions. In the long run, further combined use of the ECFL and the DMFT approaches could lead to a better understanding of the momentum-dependence of the self-energy that becomes important in lower dimensions.

The paper is organized as follows. After defining the model in Sec. [II] the general structure of the ECFL formalism is reviewed in Sec. III. In Sec. IV] we present detailed DMFT results for the hole-doped Hubbard model using high-precision Wilson's NRG as a solver. In Sec. V. the DMFT self energies are interpreted in light of the ECFL-motivated analytical expressions. The second part of the paper is devoted to the $O\left(\lambda^{2}\right)$ ECFL minimal implementation. The basic equations and their simplification in infinite dimensions are established in Sec. VI] and in Sec. VII a quantitative comparison is made to the DMFT results.

\section{MODEL}

We study the Hubbard model defined by the Hamiltonian

$$
H=\sum_{k \sigma} \varepsilon_{k} c_{k \sigma}^{\dagger} c_{k \sigma}+U \sum_{i} n_{i \uparrow} n_{i \downarrow},
$$

where $\varepsilon_{k}$ is the bare band dispersion relation obtained by Fourier transforming the hopping matrix. In this study we consider a doped Hubbard model with nearestneighbor hopping on a Bethe lattice, with semicircular density of states:

$$
\rho_{0}(\varepsilon)=\frac{2}{\pi D^{2}} \sqrt{D^{2}-\varepsilon^{2}},
$$

where $D$ is the half-bandwidth, and thus any sum over the band energy can be converted to an integral as:

$$
\frac{1}{N} \sum_{k} \mathcal{A}\left(\varepsilon_{k}\right) \rightarrow \int_{-D}^{D} d \varepsilon \rho_{0}(\varepsilon) \mathcal{A}(\varepsilon) .
$$

We note that the Fermi energy $\varepsilon_{F}$ satisfies

$$
\sin ^{-1}\left(\frac{\varepsilon_{F}}{D}\right)+\left(\frac{\varepsilon_{F}}{D}\right) \sqrt{1-\left(\frac{\varepsilon_{F}}{D}\right)^{2}}=-\frac{\pi}{2}(1-n),
$$

and vanishes near $n \sim 1$ as $\varepsilon_{F}=-\frac{\pi}{4}(1-n) D$. The hole doping level $\delta$ is related to the particle density $n$ $\left(n=N / N_{\text {sites }}\right)$ as:

$$
\delta=1-n
$$

We will use $\rho_{Q}(\omega)$ as a short-hand notation for the spectral function associated with any relevant quantity $Q\left(i \omega_{n}\right)$ (Green's function, self-energy):

$$
\rho_{Q}(\omega)=-\frac{1}{\pi} \operatorname{Im}\left(Q\left(\omega+i 0^{+}\right)\right) .
$$




\section{ECFL: GENERAL FRAMEWORK}

\section{A. ECFL formalism}

The ECFL methodology has been discussed extensively in recent literature ${ }^{2 \mid 3}$; here we highlight only the aspects that are of relevance to this work. ECFL deals with Gutzwiller-projected states obtained in the limit of $U \rightarrow \infty$, with the no-double-occupancy constraint built into the electron operators, leading to the well known $t$ - $J$ model. This results in a non-canonical theory, where familiar Feynman diagram methods fail due to the absence of Wick's theorem. The ECFL formalism is an exact alternative to the Feynman diagram technique. Instead it works with the Schwinger equations of motion for the projected electrons. It provides results for the electronic Green's functions that describe the physics of the lowenergy sector in the problem, namely the dynamics of the quasiparticle (QP) states near the Fermi energy and of the lower Hubbard band (LHB).

For our purposes, we need to express the ECFL theory in the large-dimensionality limit. The related technical problems outlined in Ref. 10 (paragraph 3) have been recently solved ${ }^{8}$ by analyzing the infinite-dimensional limit of the Schwinger equations of motion in the ECFL 11 . The exact mapping of the momentum-independent selfenergy of the infinite-dimensional Hubbard model onto that of a self-consistent Anderson impurity mode ${ }^{12}$ provides a roadmap for a suitable formulation of the ECFL equations in this limit.

In the simplest version of the ECFL theory 13 , the physical (i.e. projected) electronic Green's function is expressed as a product of an auxiliary Green's function $\mathbf{g}(k)$ and a caparison term denoted in the present work as $\widetilde{\mu}(k)$. Thus,

$$
\mathcal{G}(k)=\widetilde{\mu}(k) \times \mathbf{g}(k),
$$

where $k \equiv\left(\vec{k}, i \omega_{k}\right)$ and $\omega_{k}$ is a Fermionic Matsubara frequency. Here $\mathbf{g}(k)$ is a Fermi-liquid-like Green's function

$$
\mathbf{g}^{-1}(k)=i \omega_{k}+\mu-\left(1-\frac{n}{2}\right) \varepsilon_{k}-\Phi(k)
$$

and $\mu$ is the chemical potential. The factor $\widetilde{\mu}(k)$ (here distinguished from the chemical potential $\mu$ by the tilde), plays the role of an adaptive spectral weight, and is given by

$$
\widetilde{\mu}(k)=1-\frac{n}{2}+\Psi(k)
$$

with $\Psi(k)$ vanishing at infinite frequency. The functions $\Phi(k)$ and $\Psi(k)$ are the twin self-energies in the theory, and are exactly defined as the appropriate functional derivatives of $\mathbf{g}^{-1}$ and $\widetilde{\mu}$ respectively ${ }^{2 / 3}$. The term $\widetilde{\mu}(k)$ is termed the caparison (i.e., dressing) factor, since it provides a second layer of renormalization to the propagator $\mathbf{g}$, which is already dressed by $\Phi$. Both Green's functions satisfy an identical number sum rule $\sum_{k, \omega_{k}} \mathcal{G}(k)=\frac{n}{2}=\sum_{k, \omega_{k}} \mathbf{g}(k)$; this enables us to satisfy the Luttinger-Ward volume theorem.

In the large- $d$ limit, a further simplification can be established 8 : $\Psi$ is independent of $\vec{k}$ and $\Phi$ is decomposable into two $\vec{k}$ independent functions,

$$
\begin{aligned}
& \Psi(k)=\Psi\left(i \omega_{k}\right) \\
& \Phi(k)=\chi\left(i \omega_{k}\right)+\varepsilon_{k} \Psi\left(i \omega_{k}\right)
\end{aligned}
$$

i.e., the two frequency-dependent (but $\vec{k}$ independent) functions $\chi$ and $\Psi$ determine the Green's function.

The single-electron physical (Dyson) self-energy $\Sigma$ is defined from the single-electron Green's function $\mathcal{G}$ in the usual manner, as (using the analytic continuation $\left.i \omega_{k} \rightarrow \omega+i \eta, \eta=0^{+}\right)$:

$$
\mathcal{G}(k, \omega+i \eta)=\frac{1}{\omega+i \eta+\mu-\varepsilon_{k}-\Sigma(\omega+i \eta)} .
$$

Within the large-dimensional ECFL, the Dyson selfenergy $\Sigma$ can be related to $\Psi$ and $\chi$ as follows:

$$
\Sigma(\omega+i \eta)-\mu-\omega=\frac{\chi(\omega+i \eta)-\mu-\omega}{1-\frac{n}{2}+\Psi(\omega+i \eta)} .
$$

We see that the Dyson self-energy is manifestly momentum-independent in this limit. Note also that, as seen from (13), its real part grows linearly with $\omega$ as $\omega \rightarrow \infty$. This is a consequence ${ }^{14}$ of the Gutzwiller projection in the $U \rightarrow \infty$ limit. At finite $U$, this behavior is regularized at high-enough frequency and $\Sigma$ goes to a constant.

For a concrete implementation, the ECFL formalism allows for a perturbative expansion in a projection parameter $\lambda \in[0,1]$, ultimately identified with the double occupancy density ${ }^{3}$. The theory to $O\left(\lambda^{2}\right)$ is expected to be quantitatively accurate for densities up to $n \lesssim 0$. 715 . We postpone the description of these equations to Section VI, but note an important general insight gained from examining and evaluating such an expansion ${ }^{2|3| 15}$; the two self-energies $\chi$ and $\Psi$ have simple Fermi-liquid functional forms, with a dissipative part that is quadratic in $\omega$, at sufficiently low energies (see Figs. 11 in Sec. VI). This insight is used in the following to obtain a low-energy expansion for the Green's function.

\section{B. Low-frequency expansion of self-energies and Green's functions}

In this section we derive the low-frequency behavior of the Green's function and self-energy within the ECFL. We obtain an analytical expression which will be used to interpret and fit the DMFT results in Sec. V. We show, in particular how a characteristic particle-hole asymmetry in the Dyson self-energy is generated even when the expansion of $\operatorname{Im} \chi$ and $\operatorname{Im} \Psi$ is limited to the particle-hole symmetric lowest-order Fermi-liquid terms. 
Indeed, as mentioned above, the first few terms of a systematic $\lambda$ expansion of the ECFL equations indicate that the self-energies $\Psi$ and $\chi$ are very similar functions and resemble the self-energy of a Fermi liquid at low enough $T, \omega$, with suitable scale constants. For low $\omega$ and low $T$, up to a low-frequency cutoff scale $\Omega_{c}$, so that $|\omega| \leq \Omega_{c} \ll D$, we define (with $k_{B}=1$ )

$$
\mathcal{R}(\omega, T) \equiv \pi\left[\omega^{2}+(\pi T)^{2}\right]
$$

and write a Fermi-liquid-like expansion for the complex ECFL self-energies:

$$
\begin{aligned}
& \Psi(\omega) \sim \Psi_{0}+c_{\Psi} \omega+\frac{i}{\Gamma_{\Psi}} \mathcal{R}(\omega, T)+\Psi_{\mathrm{rem}}(\omega), \\
& \chi(\omega) \sim \chi_{0}-c_{\chi} \omega-\frac{i}{\Omega_{\chi}} \mathcal{R}(\omega, T)+\chi_{\mathrm{rem}}(\omega),
\end{aligned}
$$

where

$$
c_{\Psi}=2 \frac{\Omega_{c}}{\Gamma_{\Psi}} \quad \text { and } \quad c_{\chi}=2 \frac{\Omega_{c}}{\Omega_{\chi}} .
$$

$\Omega_{\chi}$ and $\Gamma_{\Psi}$ are parameters that determine the curvatures of the two imaginary parts, with $\Omega_{\chi}$ having the dimensions of energy, while $\Gamma_{\Psi}$ has the dimensions of the square of energy. Consequently, $c_{\Psi}$ has the dimensions of an inverse energy, while $c_{\chi}$ is dimensionless. The terms $\Psi_{\text {rem }}(\omega)$ and $\chi_{\text {rem }}(\omega)$ in Eq. (15) represent the remainders containing the leading corrections to the Fermiliquid behavior, of the type $O\left(\omega^{2}\right)$ for the real part and $O\left(\omega^{3}\right)$ for the imaginary parts of these functions. In the initial analysis below, we simply ignore these terms. They can be readily incorporated to find a systematic improvement of the fits, and lead to further corrections to the low-frequency behavior of the imaginary part of the Dyson self-energy beyond the terms considered here. Note that in Eq. (17) the constants $c_{\Psi}$ and $c_{\chi}$ also receive contributions from higher terms beyond the quadratic. Hence these approximate relations become exact if we retain the imaginary terms only to quadratic order, i.e., assuming $\rho_{\Psi} \equiv-\frac{\mathcal{R}(\omega, T)}{\pi \Gamma_{\Psi}}$ and $\rho_{\chi} \equiv \frac{\mathcal{R}(\omega, T)}{\pi \Omega_{\chi}}$. In general, however, $c_{\Psi}$ and $c_{\chi}$ can be considered as additional free parameters.

Some further remarks about this expansion are called for:

1. Expressions (15) and (16) are of the standard Fermi-liquid type (symmetric in $\omega$ for the imaginary parts). Nonetheless, when processed through the ECFL formalism, they lead to important contributions to the imaginary part of the Dysonian self-energy which are anti-symmetric in $\omega$. Revealing the origin of this important asymmetry is one of the main strengths of the ECFL analysis.
2. We shall find that as we approach half filling, $\Omega_{\chi}$ and $\Gamma_{\Psi}$ turn out to be similar functions of the electron density, in view of their parallel role in the two self-energies within the $\lambda$ expansion. In the analysis below, we will find that as $n \rightarrow 1$, it is consistent to choose $\Omega_{\chi}, \Gamma_{\Psi} \sim \delta$.

3. The energy scale $\Omega_{c}$, which determines the range of frequencies where the quadratic behavior of $\operatorname{Im} \Psi$ and $\operatorname{Im} \chi$ applies, is itself a function of the density. It shrinks linearly with $\delta=1-n$ as $n \rightarrow 1$, and therefore $c_{\Psi}$ and $c_{\chi}$ are finite as $n \rightarrow 1$. We should note that these are leading-order assumptions in $\delta=1-n$.

Thus we find at low $T, \omega$ :

$$
\begin{aligned}
& \mathcal{G}(k, \omega+i \eta) \\
& \sim \frac{\alpha_{0}+c_{\Psi} \omega+\frac{i}{\Gamma_{\Psi}} \mathcal{R}}{\omega\left(1+c_{\chi}\right)+\mu+\frac{i}{\Omega_{\chi}} \mathcal{R}-\varepsilon_{k}\left\{\alpha_{0}+c_{\Psi} \omega+\frac{i}{\Gamma_{\Psi}} \mathcal{R}\right\}},
\end{aligned}
$$

where we have introduced

$$
\alpha_{0} \equiv 1-\frac{n}{2}+\Psi_{0}
$$

and $\chi_{0}$ has been absorbed into the chemical potential $\mu$. The entire momentum-dependence is contained in $\varepsilon_{k}$. At $T=0$ and $\omega=0$ we must require $\mathcal{G}^{-1}\left(k_{F}, 0\right)=0$, so we need to set

$$
\mu=\alpha_{0} \varepsilon_{F}
$$

At low $\omega+i \eta$ and a fixed $\vec{k}$, we can write a useful expression

$$
\mathcal{G}^{-1} \sim-\varepsilon_{k}+\frac{\omega\left(1+c_{\chi}\right)+\alpha_{0} \varepsilon_{F}+\frac{i}{\Omega_{\chi}} \mathcal{R}}{\alpha_{0}+c_{\Psi} \omega+\frac{i}{\Gamma_{\Psi}} \mathcal{R}}
$$

and therefore

$$
\Sigma(\omega+i \eta) \sim \alpha_{0} \varepsilon_{F}+\omega-\frac{\omega\left(1+c_{\chi}\right)+\alpha_{0} \varepsilon_{F}+\frac{i}{\Omega_{\chi}} \mathcal{R}}{\alpha_{0}+c_{\Psi} \omega+\frac{i}{\Gamma_{\Psi}} \mathcal{R}}
$$

Note that we adjusted the self-energy so that $\operatorname{Re} \Sigma(0)=$ $\mu-\varepsilon_{F}$, thereby placing the zero-energy pole at the Fermi momentum. We now extract the wave function renormalization factor $Z$ from $Z^{-1}=\left.\frac{\partial}{\partial \omega} \mathcal{G}^{-1}(k, \omega)\right|_{\omega=0}$ as

$$
Z=\frac{\alpha_{0}}{1+c_{\chi}-\varepsilon_{F} c_{\Psi}}
$$

Using the above expansion we find the spectral function $\rho_{\mathcal{G}}(k, \omega)$ (or equivalently $A(k, \omega)$, as denoted in the exper- 
imental literature), at low $\omega$ and $k \sim k_{F}$

$$
A(k, \omega) \sim\left(\frac{\alpha_{0}^{2}}{\pi \Omega_{\Sigma}}\right) \frac{\mathcal{R}\left(1-\frac{\omega}{\Delta}\right)}{\left\{\left(1+c_{\chi}-c_{\Psi} \varepsilon_{k}\right) \omega-\alpha_{0}\left(\varepsilon_{k}-\varepsilon_{F}\right)\right\}^{2}+\left\{\alpha_{0}{ }^{2} \mathcal{R}^{2} / \Omega_{\Sigma}^{2}\right\}},
$$

where

$$
\begin{aligned}
\Omega_{\Sigma} & \equiv \alpha_{0} \frac{\Omega_{\chi} \Gamma_{\Psi}}{\Gamma_{\Psi}-\varepsilon_{F} \Omega_{\chi}}, \\
\Delta & \equiv \frac{\alpha_{0}^{2} \Gamma_{\Psi} \Omega_{\chi}}{\Omega_{\Sigma}\left\{\left(1+c_{\chi}\right) \Omega_{\chi}-c_{\Psi} \Gamma_{\Psi}\right\}} .
\end{aligned}
$$

In terms of the wave function renormalization factor $Z$,

$$
A(k, \omega) \sim\left(\frac{Z^{2}}{\pi \Omega_{\Sigma}}\right) \frac{\mathcal{R}\left(1-\frac{\omega}{\Delta}\right)}{\left\{\omega-Z\left(\varepsilon_{k}-\varepsilon_{F}\right)\right\}^{2}+\left\{Z^{2} \mathcal{R}^{2} / \Omega_{\Sigma}^{2}\right\}} .
$$

We thus obtain the following final form for the low-energy expression of the Dysonian self-energy:

$$
\begin{aligned}
& \operatorname{Im} \Sigma(\omega) \sim-\frac{\mathcal{R}}{\Omega_{\Sigma}} \frac{1-\frac{\omega}{\Delta}}{\left\{1+\omega c_{\Psi} / \alpha_{0}\right\}^{2}+\mathcal{R}^{2} /\left(\alpha_{0} \Gamma_{\Psi}\right)^{2}} \\
& \operatorname{Re} \Sigma(\omega) \sim \alpha_{0} \varepsilon_{F}+\omega \\
& -\frac{\left\{\varepsilon_{F}+\omega\left(1+c_{\chi}\right) / \alpha_{0}\right\}\left(1+\omega c_{\Psi} / \alpha_{0}\right)+\mathcal{R}^{2} /\left(\alpha_{0}^{2} \Omega_{\chi} \Gamma_{\Psi}\right)}{\left\{1+\omega c_{\Psi} / \alpha_{0}\right\}^{2}+\mathcal{R}^{2} /\left(\alpha_{0} \Gamma_{\Psi}\right)^{2}}
\end{aligned}
$$

where we recall that $\mathcal{R}(\omega, T)$ is defined in Eq. (14). These expressions, and in particular that of $\operatorname{Im} \Sigma$, are among the key results of the present paper, and will be used below in order to fit and interpret the DMFT data.

If we take the $\Psi_{\text {rem }}$ and $\chi_{\text {rem }}$ terms in Eq. $(15)$ and Eq. (16) into account, then Eq. 28) receives higher-order polynomial corrections in $\omega$ in both the numerator and denominator. Let us also note that Eq. 27) is of the form of a phenomenological version of the ECFL theory that has been recently tested against experimental data with considerable success, in some cases after adding a constant times $\left(\varepsilon_{k}-\varepsilon_{F}\right)$ in the numerator ${ }^{2617}$.

\section{Low-doping limit $n \rightarrow 1$}

At $T=0$ and near half-filling we get $\varepsilon_{F}=-\frac{\pi}{4} \delta D$ from Eq. (4). Further, from the single assumption that $\Psi_{0}=-\frac{n}{2}$ near half-filling $\frac{16}{6}$, we find that the self-energy and wave function renormalization factor scale correctly with $\delta$ as $\delta \rightarrow 0$. This assumption gives $\alpha_{0}=\delta$, and a scaling of various energy scales with $\delta$. In particular, we find from the equations that $\Omega_{\chi} \sim \Gamma_{\Psi} \sim \Omega_{c} \sim \delta$. This, together with Eq. (17), leads to $c_{\Psi} \sim O(1)$ and $c_{\chi} \sim O(1)$. This is consistent with the scaling behavior described in remarks 2 and 3 in the previous section. Keeping the dominant terms in Eqs. 223, 25, and 26, we find that

$$
\begin{aligned}
Z & =\frac{\alpha_{0}}{1+c_{\chi}}, \\
\Omega_{\Sigma} & =\alpha_{0} \Omega_{\chi}, \\
\Delta & =\frac{\alpha_{0} \Gamma_{\Psi}}{\left\{\left(1+c_{\chi}\right) \Omega_{\chi}-c_{\Psi} \Gamma_{\Psi}\right\}} .
\end{aligned}
$$

Near half-filling $(\delta \rightarrow 0)$, we define

$$
\begin{aligned}
Z & =\delta \bar{Z} \\
\Omega_{\Sigma} & =\delta^{2} \bar{\Omega}_{\Sigma}, \\
\Delta & \equiv \delta \bar{\Delta} .
\end{aligned}
$$

All objects with an overline, such as $\bar{Q}$, are determined to be finite as $\delta \rightarrow 0$. Eq. 32 is expected on general grounds near the insulating limit: to leading order $\mathcal{G}^{-1}(k, \omega)=\varepsilon_{F}-\varepsilon_{k}+\frac{\omega}{Z}+O\left(\omega^{2}\right)$, and therefore the propagating solutions correspond to quasiparticles with an energy dispersion $Z\left(\varepsilon_{k}-\varepsilon_{F}\right)$ that shrinks to zero at the insulating point $n=1$. We find here that this occurs as a linear function of $\delta$. Eq. (33), together with Eq. (28), implies that at small $\omega \sim O(\delta)$, the imaginary part of the self-energy has a finite value. Further combined with a cutoff $\Omega_{c} \sim O(\delta)$, it gives Re $\Sigma \sim \omega-2 \omega \frac{\Omega_{c}}{\Omega_{\Sigma}}$, which is then consistent with the linear vanishing of $\mathrm{Z}$ in Eq. 32. Eq. (34) shows that the particle-hole asymmetry in the spectral function increases as we approach half-filling. Finally, we see that the spectral density $\operatorname{Im} \Sigma$ becomes a scaling function of $\omega / \delta$ at low doping levels.

\section{DOPED MOTT INSULATOR: SINGLE-SITE DMFT}

The dynamical mean-field theory ${ }^{1}$ is based on the fact ${ }^{17}$ that in the limit of a large number of dimensions $d$ the self-energy becomes a momentum-independent local quantity, $\Sigma(\mathbf{k}, \omega) \rightarrow \Sigma(\omega)$. This implies that the bulk problem for $d \rightarrow \infty$ coincides with the problem of an interacting impurity embedded in an appropriate non-interacting bath 12 . DMFT formulates a practical prescription for finding this effective impurity problem and the self-consistency equation. For the Hubbard model, the corresponding impurity problem is the single-impurity Anderson model, which can be efficiently solved with the numerical-renormalization group (NRG) method 182 . 


\section{A. NRG method}

The NRG calculations have been performed with the discretization parameter $\Lambda=2$ using the discretization scheme with reduced systematic artifacts described in Ref. 22. Furthermore, the twist averaging over $N_{z}=8$ different discretization meshes has been used to reduce the oscillatory NRG discretization artifacts 23 . The truncation cutoff in the NRG was set in the energy space at $10 \omega_{N}$ (here $\omega_{N}$ is the characteristic energy at the $N$ th NRG step); such results are well converged with respect to the truncation. The $\mathrm{U}(1)$ charge conservation and $\mathrm{SU}(2)$ spin rotational invariance symmetries have been used explicitly. The raw spectral data (weighted delta peaks) were collected in bins on a logarithmic mesh with 1000 bins per frequency decade, then the broadening scheme from Ref. 24 with $\alpha=0.2$ has been used to obtain the continuous representation of the spectral functions. To calculate the self-energy $\Sigma$, we have used the procedure $e^{25}$ based on the following exact relation from equations of motion:

$$
\Sigma_{\sigma}(z)=\frac{\left\langle\left\langle\left[d_{\sigma}, H_{\mathrm{int}}\right] ; d_{\sigma}^{\dagger}\right\rangle\right\rangle_{z}}{\left\langle\left\langle d_{\sigma} ; d_{\sigma}^{\dagger}\right\rangle\right\rangle_{z}}=\frac{U\left\langle\left\langle n_{\bar{\sigma}} d_{\sigma} ; d_{\sigma}^{\dagger}\right\rangle\right\rangle_{z}}{\left\langle\left\langle d_{\sigma} ; d_{\sigma}^{\dagger}\right\rangle\right\rangle_{z}} .
$$

Here $d_{\sigma}$ is the impurity annihilation operator, while $H_{\text {int }}$ is the interaction part of the Hamiltonian. The two correlators in this expression were computed using the fulldensity-matrix NRG algorithm 2426. To accelerate the convergence of the DMFT self-consistency loop, Broyden mixing algorithm has been used 27 . This technique is particularly important to ensure the convergence at small doping as the Mott transition is approached. The Broyden solver has been used both to control the chemical potential to obtain the desired band filling and to apply the DMFT self-consistency equations 27 .

When performing the calculations in the large- $U$ limit, it is important to note that the upper Hubbard band (UHB) is outside the NRG discretization energy window. The correlator $F_{\sigma}(z)=\left\langle\left\langle n_{\bar{\sigma}} d_{\sigma} ; d_{\sigma}^{\dagger}\right\rangle\right\rangle_{z}$ receives a contribution

$$
F_{\mathrm{UHB}}(z)=\frac{w_{\mathrm{UHB}}}{z-\left(\epsilon_{d}+U\right)} \underset{U \rightarrow \infty}{\longrightarrow} \frac{w_{\mathrm{UHB}}}{-U}
$$

from the UHB, where $w_{\mathrm{UHB}}$ is the total weight of the upper Hubbard band, which in the $U \rightarrow \infty$ limit is equal to $n / 2$. The correlator $F(z)$ in Eq. (35) is multiplied by a factor $U$, thus the UHB contribution to the numerator in the $U \rightarrow \infty$ limit is equal to $-w_{\mathrm{UHB}}$. It is crucial to correct the raw numerical results by making this subtraction when the UHB is outside the discretization window, otherwise the causality is very strongly violated. (No such subtraction is necessary for the correlator $G_{\sigma}(z)=\left\langle\left\langle d_{\sigma} ; d_{\sigma}^{\dagger}\right\rangle\right\rangle_{z}$, because the UHB only makes an $\mathcal{O}(1 / U)$ contribution to the denominator.)

An analysis of the convergence of the NRG results with respect to the variation of various parameters in the method is presented in Appendix B.

\section{B. DMFT results}

\section{Scaling of quasiparticle weight $Z$ vs. doping level $\delta$}

In this work, we only consider paramagnetic solutions. At low temperatures, the DMFT equations, depending on the strength of the interaction $U$ and the doping $\delta$, give either an insulating or a metallic Fermi-liquid state. The key quantity characterizing the metallic state is the quasiparticle residue

$$
Z=\left(1-\left.\frac{\partial \operatorname{Re} \Sigma(\omega)}{\partial \omega}\right|_{\omega=0}\right)^{-1}
$$

At $\delta=0$ (half-filled system), a metallic solution is found for $U<U_{c}$ (this critical value of $U$ is often denoted $U_{c 2}$ in the DMFT literature - the spinodal of the metallic solution ${ }^{1}-$ and will be denoted $U_{c}$ here for simplicity). For $U>U_{c}$, the DMFT equations only have a unique insulating solution. At $U_{c} / D=2.918$, a Mott metalinsulator transition takes place, with characteristics similar to the Brinkman-Rice picture ${ }^{28}$ in that the quasiparticle weight $Z$ vanishes continuously and the quasiparticle effective mass diverges.

Away from half filling, i.e., for any $\delta \neq 0$, the solution is always metallic $(Z>0)$; the Mott insulator $(Z=0)$ only exists exactly at half-filling (for $U>U_{c 1}$, the spinodal of the insulator ${ }^{1}$ ). As $\delta \rightarrow 0$ for $U>U_{c}, Z$ diminishes and vanishes at $\delta=0$. This doping-driven Mott transition is illustrated in Fig. 1. In panels a-d) we plot the results of $Z$ vs. $\delta$ for a set of values of $U$. It is seen that, when considered over a broad doping range $\delta \lesssim 0.5$ (panels a and b of Fig. 11), the overall doping-dependence of $Z$ is fairly linear at intermediate values of $U / D$, while at strong coupling (large $U / D$ ) a marked curvature is seen (approximately fit by a power-law with exponent close to 1.4).

A plot of $Z / \delta$ vs. $\delta$ focusing on the low-doping region (panels $\mathrm{c}$ and $\mathrm{d}$ ) reveals, however, that the asymptotic low- $\delta$ behavior is actually linear, $Z \propto \delta$ (except close to the multicritical point $U=U_{c}, \delta=0$ where sizable corrections are found). This is indeed the behavior expected within the Gutzwiller approach28 30: Fig. 1 1 -d thus confirm that DMFT obeys this mean-field behavior. The prefactor of this linear dependence is also decreasing with $U$ in reasonable agreement with the Gutzwiller estimate $^{30} \sim\left(1-U_{c} / U\right)^{-1 / 2}$. Note that the results displayed here extend previous studies to much lower doping levels $(\delta \lesssim 0.001)$ than previously reported in the literature, due to the improvements in the NRG methodology.

\section{Self-energy and spectral function: overview of the main structures}

We now address the properties of the self-energy $\Sigma(\omega)$ and one-particle spectral function in more detail. 

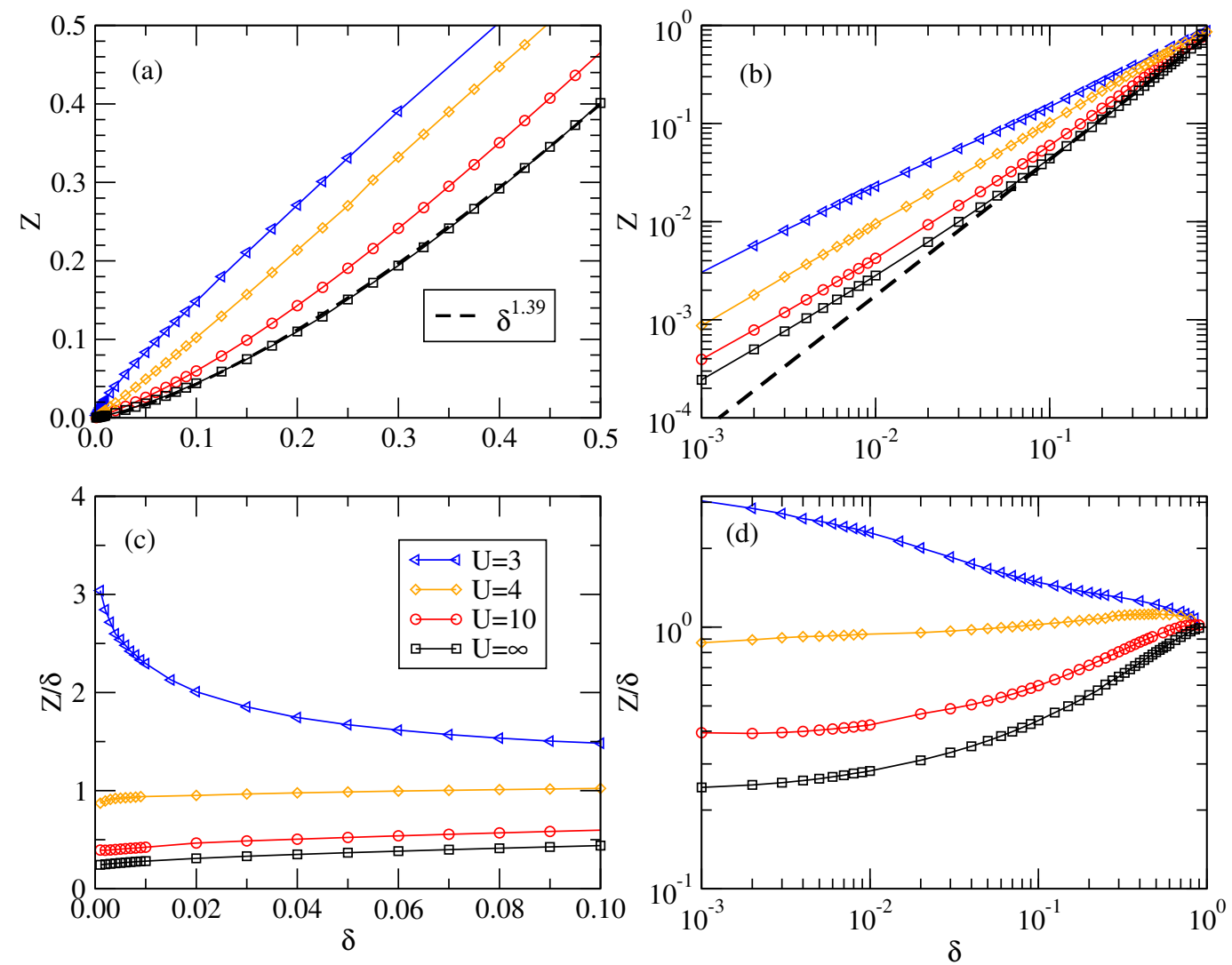

FIG. 1: (Color online) Doping-driven Mott transition within the DMFT: approach to the Mott insulating state for $U>U_{c}=$ $2.918 D$ with decreasing doping $\delta$. Top panels: doping dependence of $Z$ for a range of $\delta$ on a lin-lin (a) and on a log-log (b) plot. The dashed line is a fit to a power-law function $Z=\delta^{\alpha}$ with $\alpha=1.39$. Bottom panels: $Z / \delta$ vs. $\delta$ (c), and $Z / \delta$ vs. $\delta$ on a $\log -\log$ plot (d).

An overview plot in Fig. 2 a shows the main features in the local spectral function $A(\omega)$ and in the imaginary part of $\Sigma(\omega)$ in a broad frequency range. $\operatorname{Im} \Sigma$ has two very pronounced and sharp resonances (quasipoles). These resonances are responsible for the suppression of the spectral weight in $A(\omega)$ between the QP peak and the LHB and UHB, respectively. They are correspondingly positioned close to the minima of $A(\omega)$. In contrast to the half-filled case, where these resonances are symmetrically positioned on each side of $\omega=0$ at a scale $^{311} \propto \pm \sqrt{Z}$, their locations in the doped case are no longer symmetric and will be discussed below. In addition, there are two broad humps in $\operatorname{Im} \Sigma$ in the frequency ranges associated with the two Hubbard bands. As $U$ increases towards very large values at fixed doping, the UHB moves to higher frequencies, while the LHB and QP band gradually converge to their high- $U$ asymptotic form. This convergence is, however, rather slow and the spectra start to very closely agree with the asymptotic ones only for $U$ on the order of $100 D$.

In Fig. $2 \mathrm{~b}$ we plot a close-up on the low-energy structures, i.e., the QP band and its vicinity. We notice that the Fermi-liquid quadratic behavior of $\operatorname{Im} \Sigma$ is limited to a very narrow frequency interval, much smaller than the width of the QP peak itself. We also see (Fig. 2 and Fig. 5f that at low doping level, $\operatorname{Im} \Sigma$ develops a marked particle-hole asymmetry. These deviations to Fermiliquid behavior are discussed below in a more quantitative manner.

One of the goals of this work is to provide an analytical account of the complex frequency dependence of the self-energy that we just summarized. It should be kept in mind that the ECFL theory that we are going to use for this purpose works with the $U=\infty$ model, which begins by throwing out the UHB altogether and deals only with the LHB. Thus the comparison carried out later in this paper refers only to the LHB and QP sector (no double occupancies), containing the interesting low-energy physics of the problem.

\section{Dynamical Particle-hole asymmetry}

The local spectral function and self-energy are displayed on Fig. 3 for $U=\infty$, at two different doping levels (a small and large one, for comparison). An immediately apparent feature of these plots is the large asymmetry between hole-like $(\omega<0)$ and particle-like $(\omega>0)$ exci- 

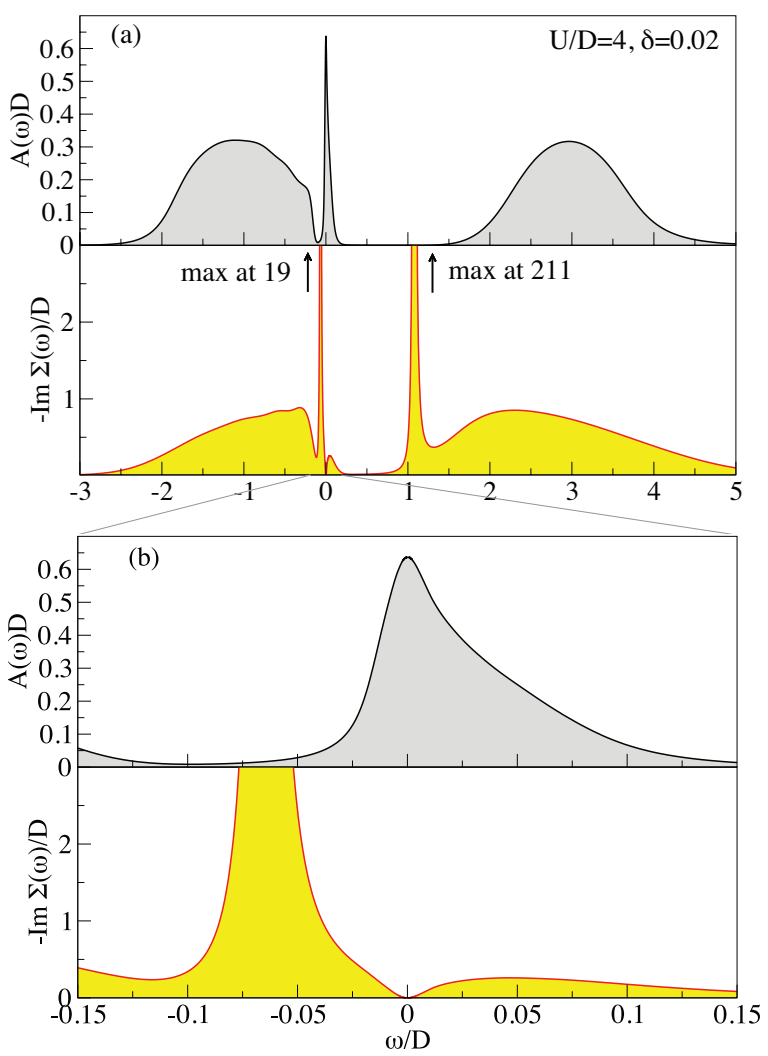

FIG. 2: (Color online) (a) Overview plot showing the full structure of spectra on high frequency scales (lower Hubbard band, quasiparticle band, upper Hubbard band) at finite $U=$ $4 D$. We show the DMFT local spectral function (top panel) and the imaginary part of the self-energy (bottom panel). (b) Close-up on the quasiparticle band at low frequencies.

tations.

Indeed, for $\omega<0,|\operatorname{Im} \Sigma|$ increases rapidly from $\omega=0$ in order to connect with the negative-energy quasi-pole. The detailed form of this increase is somewhat different depending on the doping level. At large doping it is approximately parabolic, in continuity with the low- $\omega$ FL $\sim \omega^{2}$ dependence. In contrast, at small doping, the low$\omega$ parabolic dependence evolves into a more linear-like increase at higher frequency. The local spectral function also displays an almost complete suppression of the spectral weight between the QP peak and the LHB at low doping level, while this suppression is only modest at higher doping.

In contrast, for $\omega>0,|\operatorname{Im} \Sigma|$ rapidly flattens out after its initial FL increase. It has a plateau-like behavior with a broad maximum at large and intermediate doping level (the maximum is sharper at smaller doping). Overall, $|\operatorname{Im} \Sigma|$ remains much smaller at $\omega>0$ than at $\omega<0$.

This asymmetry also reflects into the QP peak in the local spectral function $A(\omega)$, i.e., the local $\rho_{\mathcal{G}}(\omega)$, which has a very asymmetric line-shape. The decrease from its $\omega=0$ value $A(0)$ is much faster on the $\omega<0$ side, in accordance with the large $|\operatorname{Im} \Sigma|$. The detailed form of the line-shape on the more extended $\omega>0$ side is different at lower and higher doping levels, with a convex and concave shape, respectively.

Finally, the particle-hole asymmetry has a very distinctive signature in the momentum-resolved spectra $A(\varepsilon, \omega)$, which are displayed in Fig. 4. It is seen there that the dispersion of the QP peak deviates from its low-energy form $\omega_{Q P}=Z\left(\varepsilon-\varepsilon_{F}\right)$ much more rapidly on the $\omega>0$ side, where a stronger dispersion closer to that of the bare band is rapidly found. This is mostly due to the distinct behavior of the real part $\operatorname{Re} \Sigma$ for positive and negative frequencies (shown later in Fig. 6). This finding, which is also supported by the ECFL results as discussed below, is one of the main predictions of our work. It calls for the development of momentum-resolved spectroscopies for unoccupied states (the 'dark side' that is not directly accessible to ARPES). The physical significance of this 'dark side' has also been recently pointed out in cluster-DMFT studies of the two-dimensional Hubbard model ${ }^{32}$.

\section{4. $\omega / Z$ scaling}

Close to the Mott transition, all low-frequency properties are expected to scale with $Z$, i.e., be described by scaling functions ${ }^{1 / 33 / 34}$ of $\omega / Z$. This is indeed the case, as demonstrated in Fig. 5 in which good data collapse is obtained in the lowest frequency range when plotted vs. $\omega / Z$. However, we also clearly observe that the scaling is limited to the asymptotic region of very small frequencies.

On panels b and c of Fig. 5, one can compare the evolution of the shape of the local spectral function, discussed above, as a function of the doping level. One sees that the QP peak becomes increasingly asymmetric at very low doping. We also observe that the LHB has some internal structure, quite similar to that observed at half-filling as the correlation-driven Mott transition is approached from the metallic side 22135 37.

In Figs. 6, the real and imaginary part of the selfenergy are plotted against $\omega / \delta D$. The different panels cover different frequency ranges. While a better collapse of the different curves at very low frequency was obtained above when using $\omega / Z D$ as a scaling variable, it is seen from the plots in a broader frequency range that the overall structures of the self-energy obey rather good scaling properties with respect to $\omega / \delta D$. For example, the sharp peak (quasi-pole) structure at $\omega<0$ in $\operatorname{Im} \Sigma$ is seen to be located at a frequency proportional to doping level $\left(\omega_{\text {peak }} \simeq-0.7 \delta D\right)$. This peak in $\operatorname{Im} \Sigma$ is associated with the suppression of the spectral weight between the QP peak and the LHB in the spectral function. Correspondingly, it is associated with a resonance-like structure in $\operatorname{Re} \Sigma$. 

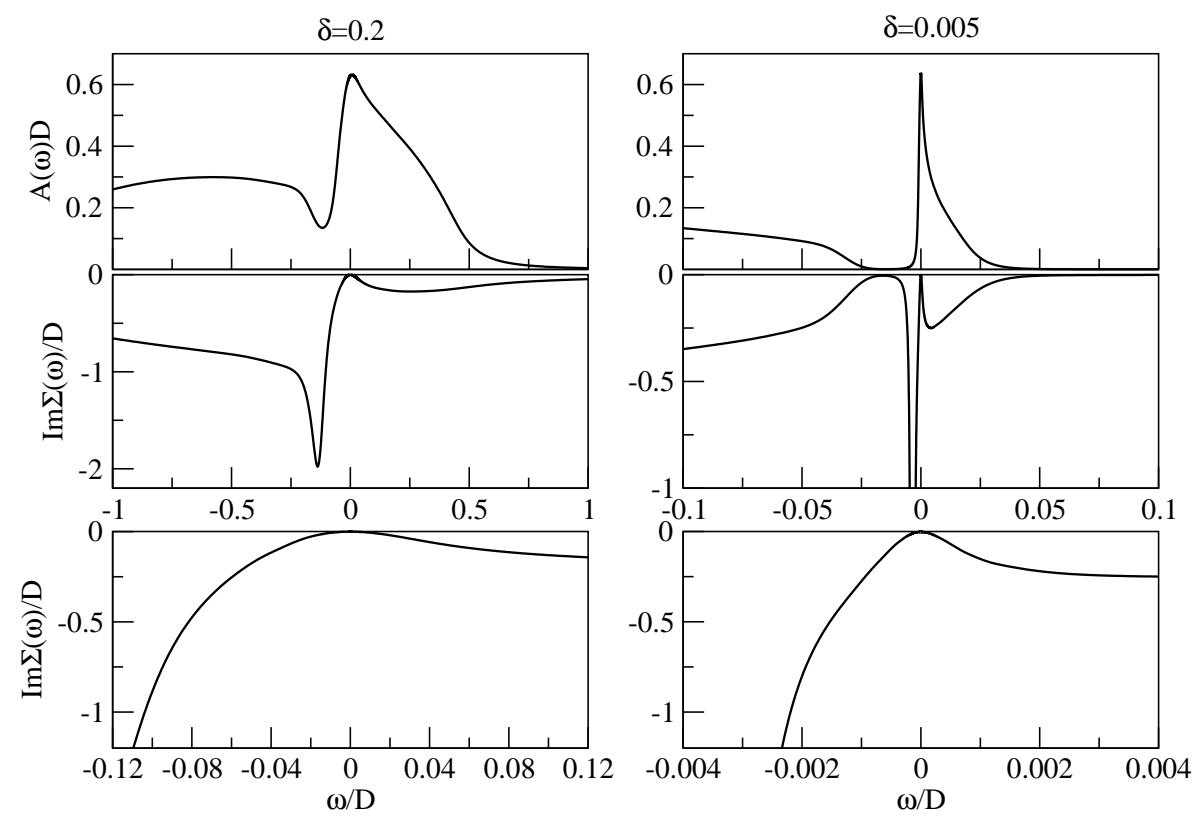

FIG. 3: (Color online) Local spectral function and the imaginary part of the self-energy for large doping $\delta=0.2$ (left) and small doping $\delta=0.005$ (right), for $U=\infty$.

\section{Deviation from the low-frequency Fermi-liquid behavior}

From panels b, c (for $\operatorname{Re} \Sigma$ ) and e, f (for $\operatorname{Im} \Sigma$ ) of Fig. 6, one can visualize the low-frequency deviations from Fermi-liquid behavior. The latter is indicated by the dashed straight and parabolic lines on this figure: $\operatorname{Re} \Sigma-\operatorname{Re} \Sigma(0)=\omega(1-1 / Z)$ and $\operatorname{Im} \Sigma \propto-(\omega / Z)^{2}$.

When visualized on an intermediate frequency scale (panels b and e) it is seen that deviation from the FL behavior is more apparent on the $\omega>0$ side, in accordance with the particle-hole asymmetry discussed above and as pointed out in previous studies ${ }^{9 / 38}$. $\operatorname{Re} \Sigma$ deviates from linearity and flattens upwards for $\omega>0$, resulting in the bending of the dispersion of $\omega>0$ quasiparticles towards the non-interacting bare dispersion, displayed above on Fig. 4. Accordingly, the deviations from parabolic behavior in $\operatorname{Im} \Sigma(\omega)$ are much more pronounced on the positive frequency side.

Zooming further on the low-frequency range (panels c and f) allows one to locate more quantitatively the deviation from the FL behavior. At $U=\infty$, it is seen to occur at $\omega_{\mathrm{FL}}^{\star} \simeq 0.1 Z D$, which is of order $0.025 \delta D$ to $0.05 \delta D$ depending on $\delta$. In agreement with previous studies 9 at finite $U$, the scale below which FL is found to apply is seen to be a very low one. It is one order of magnitude smaller than the Brinkman-Rice scale $\approx \delta D$ which corresponds to scaling the bare bandwidth by the (inverse) of the effective mass. When converted to a temperature scale, the Brinkman-Rice scale roughly corresponds to the temperature at which $\mathrm{QP}$ excitations disappear altogether (and the resistivity approaches the Mott-IoffeRegel limit) ${ }^{9}$, but it should not be identified with the much lower scale associated with deviations from FL behavior.

The low-frequency zooms in panels $\mathrm{c}$ and $\mathrm{f}$ actually reveal that the deviations from FL behavior are seen both on the $\omega<0$ and $\omega>0$ side, at similar scales $\pm \omega_{\mathrm{FL}}^{\star}$. This scale corresponds to a low-energy 'kink' in $\operatorname{Re} \Sigma$. The corresponding low-energy kink in the QP dispersion $39 \sqrt{41}$ is actually visible upon close examination of Fig. 4.

As seen on Figs. 5 b and c, the full collapse of the data is limited to very low frequencies. Two kinds of deviations from the universal behavior can be recognized. On negative frequency side, at moderate doping the deviations occur at the onset of the Hubbard band as the quasiparticle peak is not clearly separated from the LHB. On the positive frequency side, the different curves deviate from each other also in the small doping limit. Comparing the two lowest dopings, for instance, reveals the excess of the spectral weight for the lower doping curve. This suggests that the quasiparticle peak is not fully characterized by the renormalization factor $Z$ alone: the quasiparticle peak weight $W_{\text {q.p. }}$ and $Z$ are not necessarily simply proportional. This question is best addressed at very low 

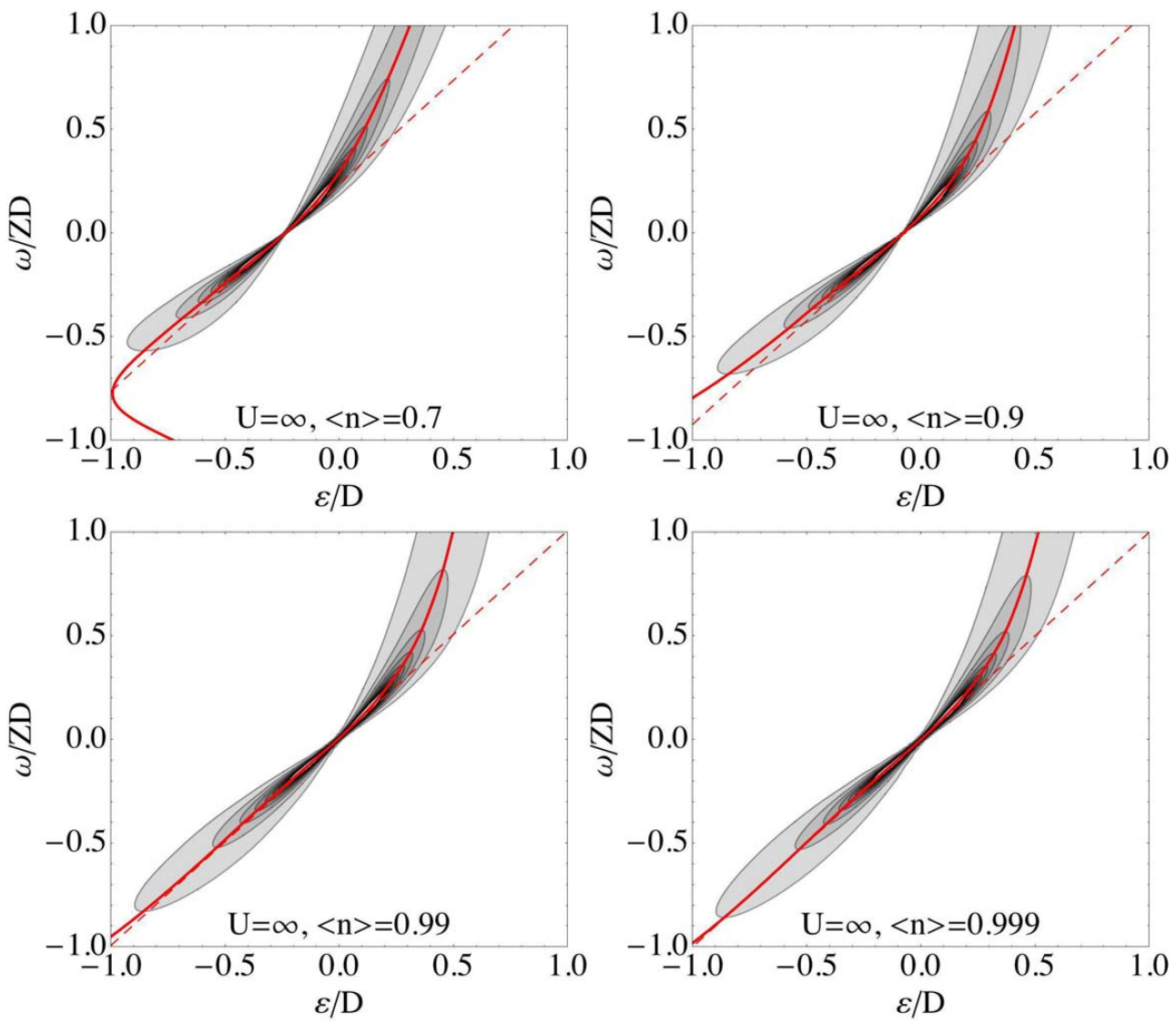

FIG. 4: (Color online) Intensity plots of the momentum $\left(\varepsilon_{k^{-}}\right)$resolved spectral function $A(\varepsilon, \omega)$ for $U=\infty$ at four different doping levels, plotted as a function of $\varepsilon / D$ and $\omega / Z D$. The plain line locates the solution of the QP pole equation $\omega+\mu-$ $\operatorname{Re} \Sigma(\omega)-\varepsilon=0$ (neglecting $\operatorname{Im} \Sigma$ ). By definition of the QP excitations, this line has slope unity (cf. dashed line) at low- $\omega$ when plotted in this manner since $\omega_{\mathrm{QP}}=Z\left(\varepsilon-\varepsilon_{F}\right)$ (i.e., $\left.v_{F}^{\star}=Z v_{F}\right)$ within the DMFT.

doping, when the quasiparticle peak is well separated from the LHB. We can then extract $W_{\text {q.p. }}$ by integrating the spectral function between the two local minima in $A(\omega)$. The results are plotted in Fig. 5 $\mathrm{d}$. We find that at low $\delta, W_{q . p}$. and $Z$ are related by a power-law $W_{\text {q.p. }}=Z^{\gamma}$, with $\gamma$ close to one, but not exactly 1 . More specifically, $\gamma$ is found to be $U$-dependent: $\gamma=1.017$ for $U=3, \gamma=1.039$ for $U=4, \gamma=1.049$ for $U=10$ and $\gamma=1.067$ for $U=\infty$.

\section{Charge compressibility: absence of phase separation}

For some types of the (non-interacting) conductionband density of states, there can be phase separation near half filling 42 . We verify that this is not the case for the Bethe lattice by plotting the band filling $n$ as a function of the chemical potential $\mu$ in Fig. 7. panel a) for $U=$ $\infty$. The dependence is monotonous, thus all solutions are physically stable with positive charge compressibility $\kappa=\partial n / \partial \mu$. We also plot the quasiparticle residue $Z$ as a function of the chemical potential $\mu$ (panel b). The charge compressibility $\kappa$ as a function of the band filling (panel c) has a maximum near quarter filling. For smaller $n$, the decrease is due to the particular form of the noninteracting DOS (semi-circular function). For larger $n, \kappa$ drops to zero as the Mott transition is approached. The asymptotic behavior is a power law $\delta^{\beta}$ with $\beta \approx 1 / 5$.

\section{DOPED MOTT INSULATOR: AN ECFL PERSPECTIVE ON THE DMFT}

In this section we make use of the general structure of the self-energy resulting from the ECFL in order to interpret, fit, and better understand the complex frequencydependence of the DMFT self-energies. The emphasis will be on the intermediate frequency range which encompasses both the vicinity of $\omega=0$ and of the quasipole (sharp peak) in $\operatorname{Im} \Sigma$ on the negative frequency side at $\omega_{\text {peak }} \simeq-0.7 \delta D$. We focus on intermediate doping levels which turns out to be the range where the ECFL applies best, rather than on very low doping. For these reasons, we can use as a scaling variable:

$$
x \equiv \omega / \delta
$$



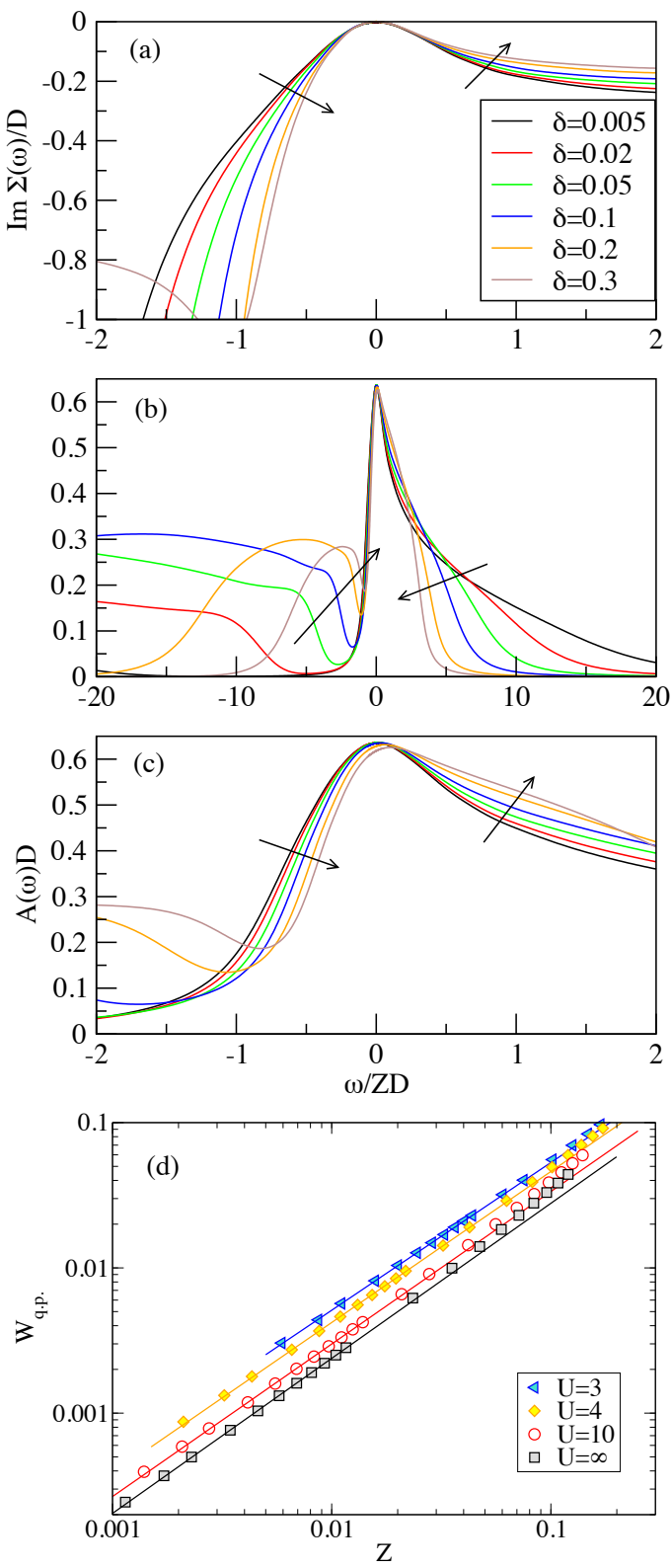

FIG. 5: (Color online) (a) Imaginary part of the self-energy $\Sigma$ versus the rescaled frequency $\omega / Z$ for $U=\infty$. (b,c) Corresponding local spectral functions. Note that when plotted vs. $\omega / Z$, the peak related to the onset of the LHB moves to the left with diminishing $\delta$, as seen in panel b. The results for finite $U$ are qualitatively very similar. The arrows indicate the direction of the increasing value of $\delta$.

\section{A. ECFL line-shapes: main features}

The low frequency ECFL analysis from Sec. II gives a simple expression, Eq. (28), for $\operatorname{Im} \Sigma$ at $T=0$. Using the overline convention of Eqs. (32 33, 34) to denote variables that remain finite as $\delta \rightarrow 0$, e. g. $P=P / \delta$, we rewrite
Eq. 28 as

$$
\operatorname{Im} \Sigma=-\frac{x^{2}}{\bar{\Omega}_{1}} \frac{1-x / \bar{\Delta}_{1}}{\left(1+x / \bar{\Delta}_{2}\right)^{2}+x^{4} / \bar{\Omega}_{2}^{2}} .
$$

This ansatz function is determined by two variables with the meaning of curvature $\bar{\Omega}_{1,2}$ that are simply related to $\Omega_{\Sigma}$ and $\Gamma_{\Psi}$, respectively, and by two parameters which adjust the asymmetry $\bar{\Delta}_{1,2}$ that are related to $\Delta$ and $c_{\psi}$.

The numerator of the expression describes a parabolic dependence, with a cubic correction term. This ansatz function has a peak (quasi-pole) at frequency $x=-\bar{\Delta}_{2}$ for a finite $\bar{\Omega}_{2}$, turning into a true pole when $\bar{\Omega}_{2} \rightarrow \infty$. The low-frequency asymmetry of the self-energy, important for the low-temperature thermoelectric properties, arises through a combination of the terms present in the numerator and denominator. Expanded to cubic order in frequency, the ansatz gives

$$
\operatorname{Im} \Sigma=-x^{2} / \bar{\Omega}_{1}\left[1-\left(1 / \bar{\Delta}_{1}+2 / \bar{\Delta}_{2}\right) x\right] .
$$

The ansatz function and its evolution as the parameters are varied is illustrated in Fig. 8 .

Summarizing, the ansatz function Eq. 39 contains a parabolic dependence, multiplied by a function with a sharp peak at negative frequencies; therefore, it can be expected to describes the coarse structure found in the DMFT very well already at this order including only the Fermi-liquid structure of the underlying functions $\Psi$ and $\chi$.

\section{B. ECFL fits of the DMFT self-energy}

The DMFT results for $U=\infty$ self-energy for a range of doping levels are presented in Fig. 9 together with fits to Eq. (39). At large doping, the ansatz function describes the DMFT data remarkably well: the low-frequency dependence and the main shape of the self-energy are fully reproduced.

At smaller doping, the quasi-pole at negative frequencies becomes very sharp and $\operatorname{Im} \Sigma$ in DMFT develops a "nipple-like" structure at low-frequency, with semi-linear frequency dependence at negative frequencies. These two features (quasi-pole and nipple) cannot be simultaneously well described by the simplest ECFL ansatz function in a broad frequency range. Given that the ansatz has a structure that already contains the pole, the fits in a broad frequency window are more meaningful. The DMFT data can still be described successfully, but terms beyond the lowest-order Fermi-liquid form in $\Psi$ and $\chi$ need to be retained. Work along these lines to reproduce the precise shape and to analyze its physical contents should be possible.

The evolution with doping of the fitting parameters is shown in Fig. 10. The first observation is that the fitting parameters (except for $\bar{\Delta}_{1}$ to be discussed below) do not depend substantially on the doping, hence validating our assumptions stated above. 

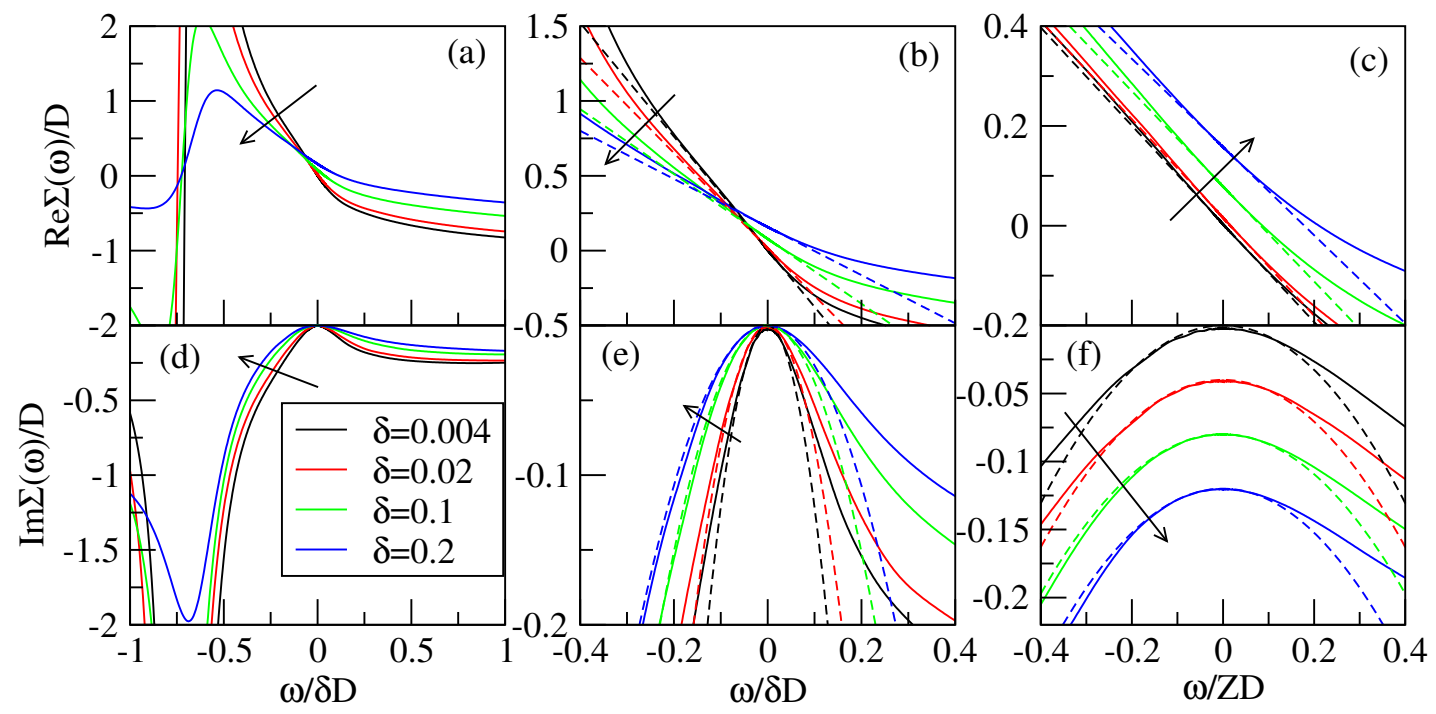

FIG. 6: (Color online) Real and imaginary parts of the DMFT self-energy $\Sigma(\omega)$ for a range of doping for $U=\infty$, as a function of $\omega / \delta D$ (left and centre) or as a function of $\omega / Z D$ (right). The arrows indicate the direction of the increasing value of $\delta$. The linear (for real parts) and parabolic (for imaginary parts) are performed in the frequency range $[-0.05: 0.05] \delta D$. In panel (f), the data is vertically offset for clarity.
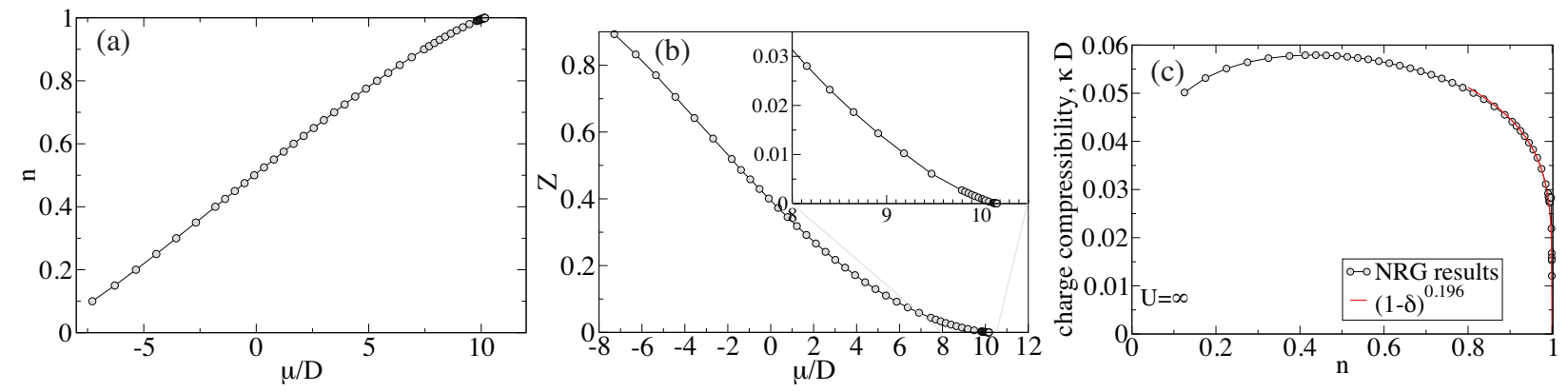

FIG. 7: a) Band filling $n$ vs. chemical potential $\mu$ for $U=\infty$. b) Quasiparticle residue $Z$ vs. chemical potential $\mu$ for $U=\infty$. c) Charge compressibility for $U=\infty$.

The second observation is that $\bar{\Omega}_{1}$ is usually found to be very close to $\bar{\Omega}_{2}$. This supports the conclusions of the $\lambda^{2}$ analysis (to be discussed in the next section) which also finds that $\Gamma_{\Psi}$ is close to $\Omega_{\chi}$.

The third observation is that the bulk of the asymmetry does actually not come from the explicitly cubic term (parametrized by $1 / \Delta_{1}$ ), but rather from $1 / \Delta_{2}$. Hence, it is the presence of the quasi-pole at negative frequency which is responsible for the strong particlehole asymmetry. This is seen most explicitly in the doping range $\delta=0.2-0.3$, where $1 / \Delta_{1}$ almost vanishes and where, furthermore, the data is excellently described by the ansatz function from Eq. (39). The physical content of this observation might be that the lowfrequency particle-hole asymmetry is (at least at not too low dopings) directly related to the presence of the LHB at much higher frequency scales, and thus ultimately to the strong-correlation physics. This observation is consistent with the picture emerging from the ECFL, where the asymmetry is a consequence of the Gutzwiller pro- jection.

\section{Summary}

To summarize, the ECFL-derived ansatz, Eq. (39), which retains only the lowest-order Fermi-liquid terms in $\Psi$ and $\chi$, describes the rather complex frequency dependence of the DMFT data remarkably well at low to intermediate frequencies and for not too small doping levels. Importantly, retaining only the Fermi-liquid (hence particle-hole symmetric) terms in these ECFL self-energies already yields a marked particle-hole asymmetry in the physical electron Dysonian self-energy. The ansatz also describes the pole at negative frequencies, associated with the onset of the LHB. Whereas the Fermiliquid behavior only applies at extremely low frequencies in the Dysonian self-energy, the Fermi-liquid concepts can still be used over a much broader frequency range when proper auxiliary quantities are considered, within 

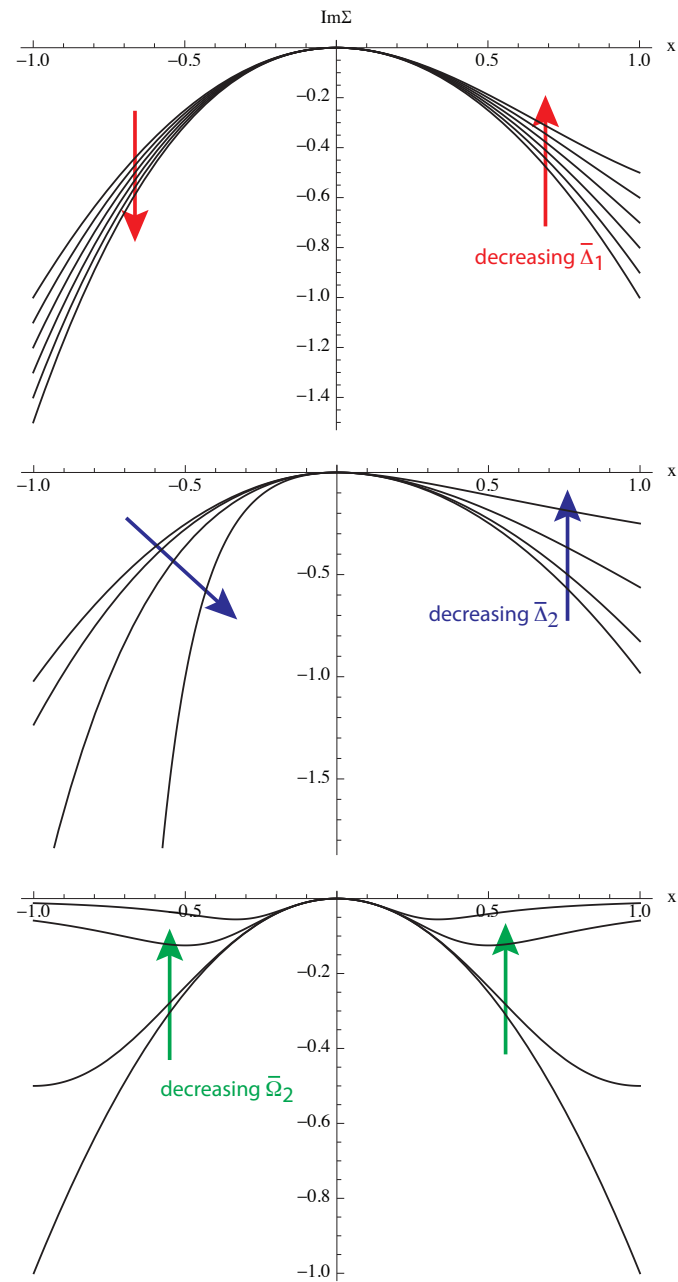

FIG. 8: (Color online) Role of the parameters in the ECFL ansatz function, Eq. (39), use for fitting in Figs. 9 and 10

the broader framework provided, e.g., by the ECFL theory. At lower doping levels, however, the DMFT results display structures (nipple) which signal the increasing importance of corrections beyond the dominant Fermiliquid terms in $\Psi, \chi$.

\section{ECFL: EXPANSION TO $O\left(\lambda^{2}\right)$}

\section{A. Summary of equations}

We now summarize the results of the $O\left(\lambda^{2}\right)$ expansion of the ECFL equations in Ref. 8, which are then computed and compared with the DMFT results. We note that the ECFL reformulation of the Dyson selfenergy $\Sigma(\omega)$ into the ECFL auxiliary self-energies $\Psi(\omega)$ and $\chi(\omega)$ is exact. Therefore, if one could perform the $\lambda$ expansion to infinite-order in $\lambda$, one would obtain the exact answer for these auxiliary self-energies, and consequently the Dyson self-energy. The resulting Dyson-self energy would then agree exactly with the one obtained through DMFT for the case of infinite-U. Our aim here is to benchmark the lowest non-trivial order of the $\lambda$ expansion against the exact DMFT results. Note that in the $d \rightarrow \infty$ limit, in the paramagnetic phase, the singleparticle properties of the $t-J$ model are identical to those of the $U=\infty$ Hubbard model. In other words, as long as antiferromagnetic correlations are short-ranged, $J$ does not enter single-particle properties in the $d=\infty$ limit. Accordingly, the only coupling constant entering the simplified ECFL equations is the hopping (band dispersion) itself, and not the superexchange.

In the $O\left(\lambda^{2}\right)$ scheme, the explicit density factors $1-\frac{n}{2}$ that occur in Eq. (8) and (9) are replaced by the rule

$$
1-\frac{n}{2} \rightarrow a_{\mathcal{G}} \equiv 1-\lambda \frac{n}{2}+\lambda^{2} \frac{n^{2}}{4}+O\left(\lambda^{3}\right) .
$$

The second rule is that the explicit self-energy expressions in these equations are multiplied by $\lambda$. As an illustration of these rules, we write Eq. (8) and (9) as

$$
\begin{aligned}
\mathbf{g}^{-1}(k) & =i \omega+\mu-a_{\mathcal{G}} \varepsilon_{k}-\lambda \Phi(k), \\
\widetilde{\mu}(k) & =a_{\mathcal{G}}+\lambda \Psi(k) .
\end{aligned}
$$

The factor $\lambda$ is set to 1 before actually computing with these formulas. The two self-energy functions $\Phi(k)$ and $\Psi(k)$ satisfy the equations to second order in $\lambda$ :

$$
\begin{aligned}
\Psi\left(i \omega_{k}\right)= & -\lambda \sum_{p q}\left(\varepsilon_{p}+\varepsilon_{q}-u_{0}\right) \mathbf{g}(p) \mathbf{g}(q) \mathbf{g}(p+q-k), \\
\Phi(k)= & \left(\varepsilon_{k}-\frac{u_{0}}{2}\right) \Psi\left(i \omega_{k}\right)+\chi\left(i \omega_{k}\right)-u_{0}\left(\lambda \frac{n^{2}}{8}-\frac{n}{2}\right) \\
& -\sum_{p} \varepsilon_{p} \mathbf{g}(p) \\
\chi\left(i \omega_{k}\right)= & -\lambda \sum_{p q}\left(\varepsilon_{p+q-k}-\frac{u_{0}}{2}\right)\left(\varepsilon_{p}+\varepsilon_{q}-u_{0}\right) \times \\
& \mathbf{g}(p) \mathbf{g}(q) \mathbf{g}(p+q-k) .
\end{aligned}
$$

All equations in Eq. (44) are implicitly understood to have $O\left(\lambda^{3}\right)$ corrections, so that the $\mathbf{g}$ and $\widetilde{\mu}$ pieces of $\mathcal{G}$ in Eq. (7) are correct to the stated order. As expected, the functions $\Psi, \chi$ depend on the frequency but not the momentum $\vec{k}$. Both Green's functions satisfy an identical number sum rule $\sum_{k, \omega_{n}} \mathcal{G}(k)=\frac{n}{2}=\sum_{k, \omega_{n}} \mathbf{g}(k)$, and the theory has two chemical potentials necessary to impose these, namely $\mu$ and $u_{0}$. As discussed in Ref. 3 , the second chemical potential $u_{0}$ arises from the requirement of satisfying a "shift invariance" in the theory. The shift transformation in the present model acts as $\varepsilon_{p} \rightarrow \varepsilon_{p}+c$. This transformation shifts the center of gravity of the band; it is absorbable in $u_{0}$, and thus rendered inconsequential. We can easily verify that $\chi$ and $\Psi$ are independently shift-invariant. Combining the expressions, we write

$$
\begin{aligned}
\mu^{\prime} & \equiv \mu+u_{0}\left(\lambda^{2} \frac{n^{2}}{8}-\lambda \frac{n}{2}\right)-\frac{u_{0}}{2} a_{\mathcal{G}}+\lambda \sum_{p} \varepsilon_{p} \mathbf{g}(p), \\
\mathbf{g}^{-1}(k) & =i \omega+\mu^{\prime}-\left(\varepsilon_{k}-\frac{u_{0}}{2}\right)\left\{a_{\mathcal{G}}+\lambda \Psi\left(i \omega_{k}\right)\right\}-\lambda \chi\left(i \omega_{k}\right) .
\end{aligned}
$$




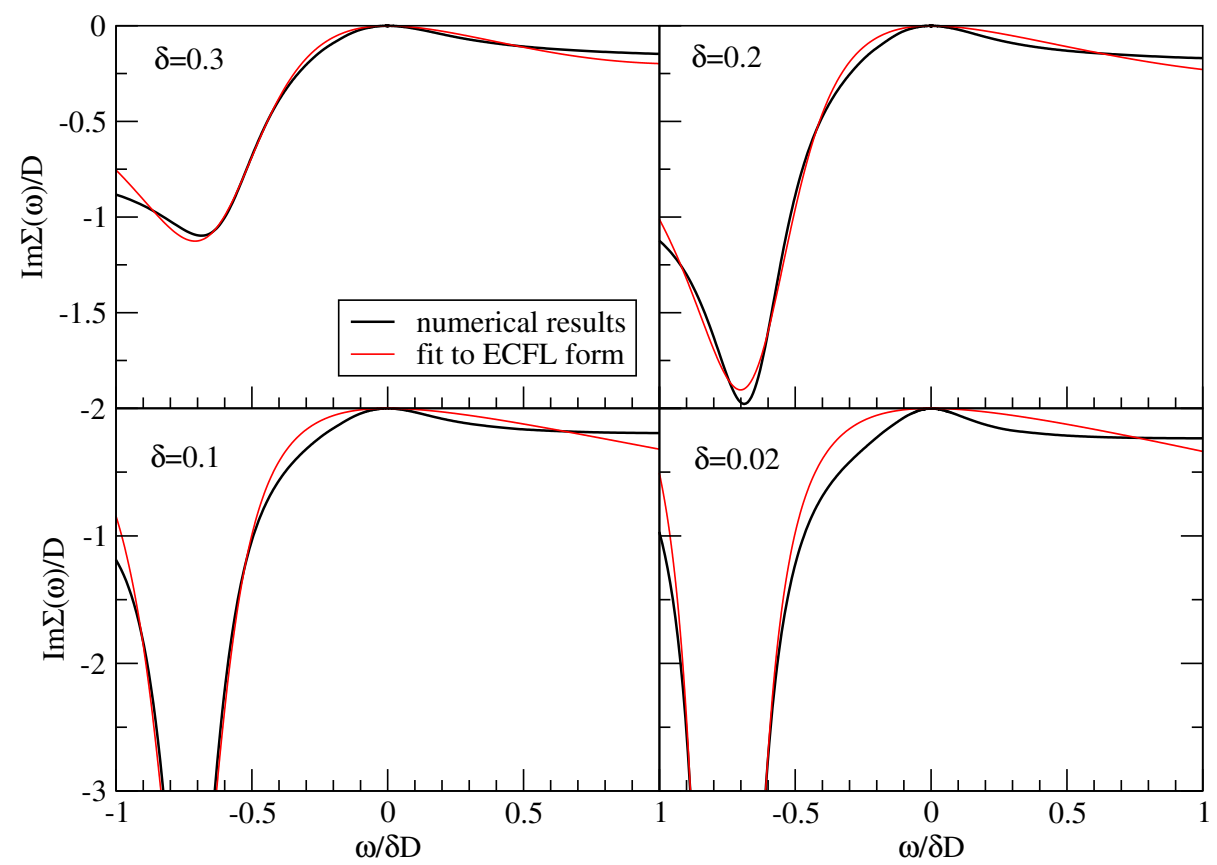

FIG. 9: (Color online) $\operatorname{Im} \Sigma(\omega)$ vs. rescaled frequency $\omega / \delta D$ and fits to Eq. $(39)$ for $U=\infty$. The ansatz describes the DMFT self-energy at moderate dopings remarkably well. The nipple structure that becomes pronounced at small doping signals that $\chi$ and $\Psi$ develop non-Fermi-liquid corrections.

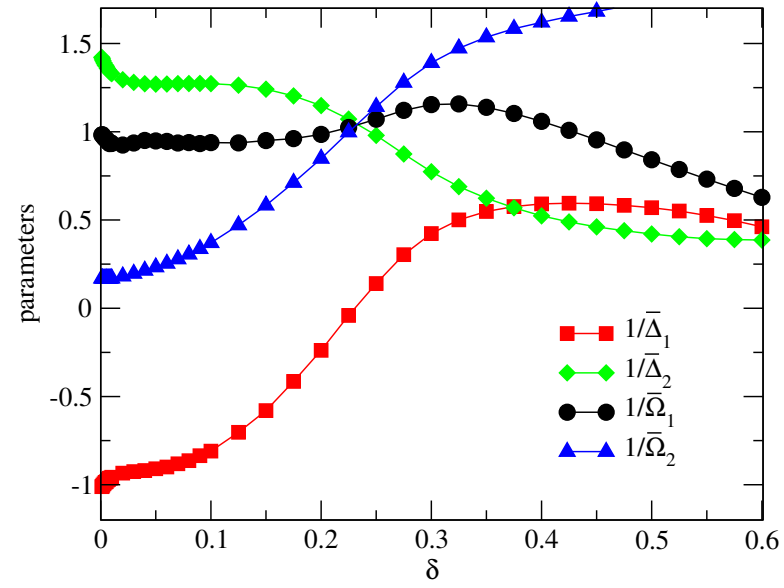

FIG. 10: (Color online) Parameters in the fit function, Eq. (39), for a range of doping $\delta$ at $U=\infty$. For a broad fitting energy range, the fitting parameters are smooth as a function of $\delta$, thus the fitting procedure is well defined. At dopings where the fits work best (that is around $\delta=0.25$ ), $\bar{\Omega}_{1}$ is found to be close to $\bar{\Omega}_{2}$.

The Green's function is then found by combining Eq. (45), (44) and (43) in the expression Eq. (7).

\section{B. Setting up the computation}

To set up the computation, we write a local Green's function with weight $m=0,1, \ldots$ using Eq. (45) as

$$
\begin{aligned}
& \mathbf{g}_{l o c, m}\left(i \omega_{k}\right) \equiv \sum_{\vec{k}} \mathbf{g}(k)\left(\varepsilon_{\vec{k}}\right)^{m} \\
= & \int_{-D}^{D} d \varepsilon \rho_{0}(\varepsilon) \frac{\varepsilon^{m}}{i \omega+\mu^{\prime}-\left(a_{\mathcal{G}}+\Psi\right)\left(\varepsilon-\frac{u_{0}}{2}\right)-\chi},
\end{aligned}
$$

where $\chi$ and $\Psi$ are functions of frequency $i \omega_{k}$ but not the energy $\varepsilon$. We find that both $\mathbf{g}_{l o c, 0}$ and $\mathbf{g}_{l o c, 1}$ are needed to compute the frequency-dependent self-energy. Similarly, a local $\mathcal{G}$ can be defined, and the number sum rules can be written as $\frac{n}{2}=\sum_{i \omega} \mathbf{g}_{l o c, 0}(i \omega)$ and $\frac{n}{2}=\sum_{i \omega} \mathcal{G}_{l o c, 0}(i \omega)$. Where necessary, the usual convergence factor $e^{i \omega 0^{+}}$is inserted. The two $\vec{k}$ independent functions $\Psi$ and $\chi$ in Eq. (44) can be written in a compact way if we first define a function with three indices $\left(m_{1} m_{2} m_{3}\right)$ from the weight factors:

$$
\begin{aligned}
& I_{m_{1} m_{2} m_{3}}(i \omega)=-\frac{1}{\beta^{2}} \sum_{\nu_{1}, \nu_{2}} \mathbf{g}_{l o c, m_{1}}\left(i \nu_{1}\right) \times \\
& \mathbf{g}_{l o c, m_{2}}\left(i \nu_{2}\right) \mathbf{g}_{l o c, m_{3}}\left(i \nu_{1}+i \nu_{2}-i \omega\right) .
\end{aligned}
$$

After continuation $i \omega \rightarrow \omega+i \eta$, and for all values of the indices, the low frequency and temperature $I(i \omega)$ is a Fermi-liquid-like self-energy with an imaginary part $\propto$ 
$\left(\omega^{2}+\left(\pi k_{B} T\right)^{2}\right)$. We can now rewrite Eq. 44 as:

$$
\begin{aligned}
& \Psi(i \omega)=-u_{0} I_{000}(i \omega)+2 I_{010}(i \omega) \\
& \chi(i \omega)=-\frac{u_{0}}{2} \Psi(i \omega)-u_{0} I_{001}+2 I_{011}(i \omega) .
\end{aligned}
$$

Clearly, Eqs. (48) and (46) along with the definition (47) and the number sum rules form a self-consistent set of equations that can be solved iteratively on a computer. The Dyson self-energy and the spectral function can be computed in terms of these quantities using Eq. (13)

\section{Auxiliary and Dyson self energies to $O\left(\lambda^{2}\right)$}

In Fig. 11 we present $\rho_{\chi}, \rho_{\psi}$, and $\rho_{\Sigma}$ (from top to bottom). $\rho_{\psi}$ and $\rho_{\chi}$ have similar frequency-dependence and a Fermi-liquid form is obeyed much more accurately than what is found for the Dyson self-energy $\rho_{\Sigma}$. This supports the ansatz that we employed above. In particular, the auxiliary self energies are more particle-hole symmetric; most of the particle-hole asymmetry follows from the structure of ECFL equations. This signals that the Fermi liquid concept has validity outside of the canonical Fermi liquid behavior.

\section{DETAILED COMPARISON OF $O\left(\lambda^{2}\right)$ ECFL RESULTS TO DMFT}

\section{A. The effective density of the ECFL spectral functions and its phenomenological adjustment}

The $O\left(\lambda^{2}\right)$ equations of ECFL discussed here give a high- $\omega$ limiting behavior $\mathcal{G} \sim \frac{a_{\mathcal{G}}}{\omega}$, differing from the exact form $\mathcal{G} \sim \frac{1-\frac{n}{2}}{\omega}$ due to the replacement $1-\frac{n}{2} \rightarrow a_{\mathcal{G}} \equiv$ $1-\lambda n / 2+\lambda^{2} n^{2} / 4$ as per the rules of the calculation. This effect is due to the incomplete projection of the $O\left(\lambda^{2}\right)$ treatment of the ECFL equation of motion. At $n \sim 0.75$ the error in the high-frequency weight is $22.5 \%$.

A phenomenological scheme for adjusting for this feature defines an effective density $n_{\text {eff }}$, using the ratio of particle addition and removal states as the relevant metric, so that $\frac{n}{1-n+n^{2} / 4}=\frac{n_{\text {eff }}}{1-n_{\text {eff }}}$, thus yielding

$$
n_{\mathrm{eff}}=\frac{n}{1+\frac{n^{2}}{4}} .
$$

Clearly higher order calculations would have a corresponding mapping between the two densities. For several of the comparisons below, agreement is greatly improved by plotting the results of ECFL as a function of $n_{\text {eff }}$.

\section{B. Comparison between $O\left(\lambda^{2}\right)$-ECFL and DMFT}

We find that the computed values of the quasiparticle weight $Z$ from ECFL are close to the $U / D=4$ DMFT
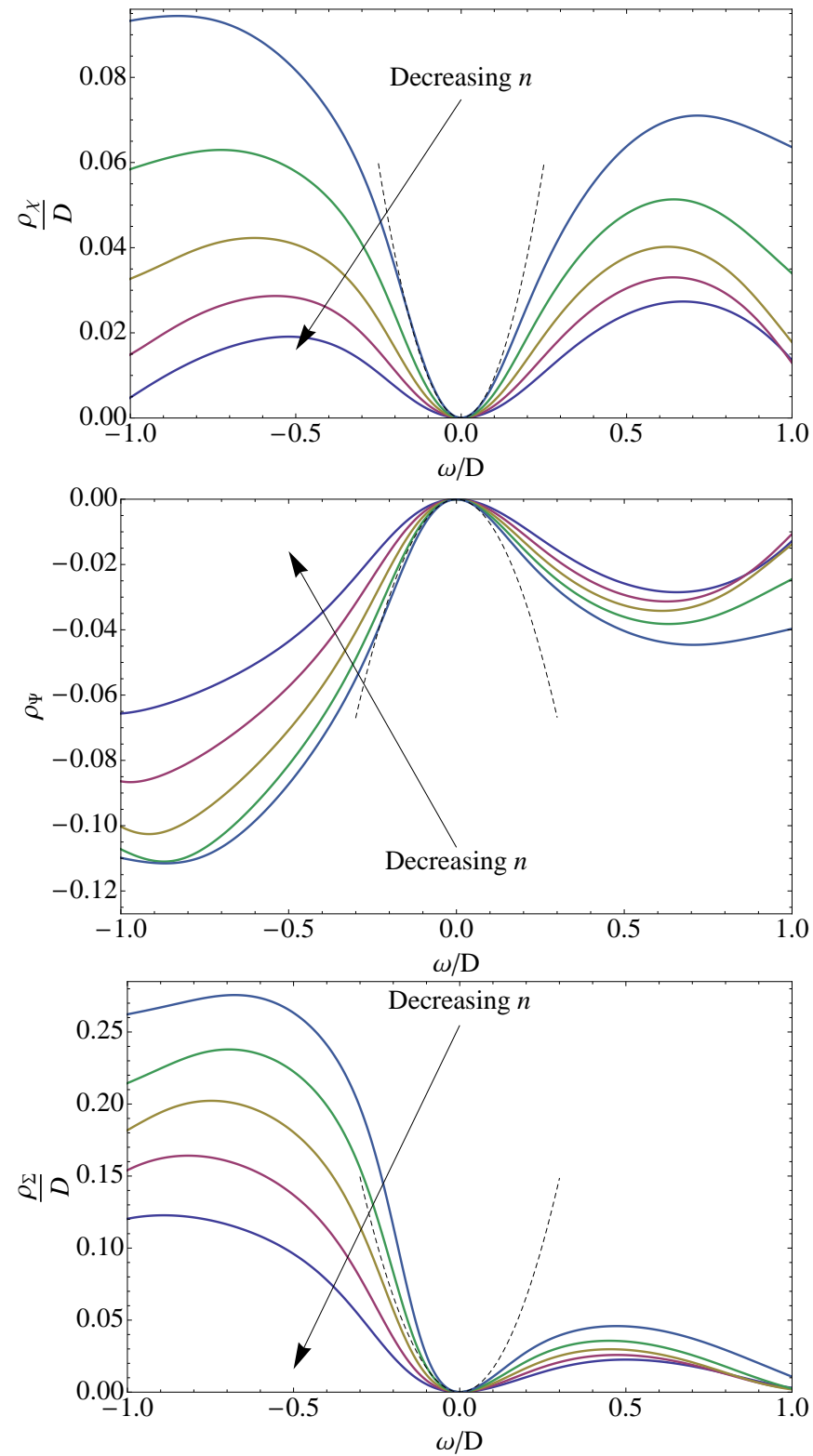

FIG. 11: (Color online) Imaginary parts (spectral densities) of the auxiliary self-energies $\chi$ and $\Psi$, and the Dyson self-energy $\Sigma$ within the $O\left(\lambda^{2}\right)$ ECFL. The dotted lines are parabolic fits at the highest density. Recall that typical Fermi-liquid-type spectral functions exhibit a parabolic and therefore particle-hole symmetric behavior over a large energy range. From these fits one observes that the auxiliary functions $\Psi$ and $\chi$ have a Fermi-liquid form over a wider energy range than the Dyson self-energy $\Sigma$.

curve, we detail this in the Appendix A, where the momentum distribution is also shown. This is suggestive of an analogy between the two incompletely projected theories. In particular, making $U$ finite, and truncating the $\lambda$ expansion at second order, both introduce some double occupancy into the system. It is therefore not surprising that the $U / D=4$ DMFT results agree better with the $O\left(\lambda^{2}\right)$-ECFL than with the $U / D=\infty$ DMFT results. 
However the limitations of the $O\left(\lambda^{2}\right)$ calculation within ECFL preclude obtaining reliable results for doping levels smaller than $\delta \approx 0.25$.

\section{Spectral lineshapes}

In Fig. 12 we compare the ECFL and the DMFT results at $U=4 D$ and $U=\infty$ for the $\epsilon$-resolved spectral functions at two values of the band energy, $\varepsilon_{k}=-D$ and $\varepsilon_{k}=\varepsilon_{F}$. In general, the agreement is encouraging. At $\varepsilon_{k}=-D$ (left panel), DMFT has a deeper minimum between the QP and the secondary feature at high binding energy than is seen in ECFL, but the position of the ECFL peaks agrees well with that of the DMFT peaks. At $\varepsilon_{F}$ (right panel) the QP are of similar width but have different values of $Z$, as discussed above. The background of width $\sim D$ lies over essentially the same frequency range for all three calculations, and has a peak at $\omega=-0.5 D$, approximately the same position for each data set. However, the height of the peak is less pronounced for the ECFL than the DMFT. At positive frequencies the spectral functions are in excellent agreement. Plotting the spectral function as a function of the scaled frequency $\omega / Z D$ improves the agreement in the position and width of the quasiparticle, as illustrated in the more sensitive self-energy curves in Fig. 13. We note that the scaled ECFL curves agree well with the DMFT curves even for density $n \sim 0.8-0.9$ for scaled frequency $|\omega| \leq 0.5 \mathrm{DZ}$. We find this agreement surprising in view of our criterion discussed above, placing $n \sim 0.75$ as the limiting density.

The physical spectral function $A(\omega)$, when displayed as a color intensity plot using the scaled frequency $\omega / Z D$ as in Fig. 14, further emphasizes this similarity. At this level of description, the $U=4 D$ DMFT curve and the $O\left(\lambda^{2}\right)$ calculation look almost identical. In particular, as clear from this figure, both theories indicate that the quasiparticle peak becomes rapidly more dispersive as one moves to positive energies, corresponding to unoccupied states (i.e., the effective Fermi velocity increases as compared to its low-energy value and becomes closer to the band value). As discussed above (Sec. IV] Fig. 44, this is one of the primary common conclusions of both theories, which could be tested in future experiments able to probe the unoccupied states in a momentum-resolved manner.

In view of the remarkable similarity between the different theories, as seen in Fig. 13 and Fig. 14, it appears that the $O\left(\lambda^{2}\right)$ version of ECFL has the correct shape of the spectra built into it, but requires a correction for a too large value of the QP factor $Z$. This is the main conclusion of this work regarding the benchmarking of the ECFL.

\section{CONCLUSION AND PROSPECTS}

In this work we have presented a detailed comparison between the DMFT and the ECFL theories, applied to the doped Hubbard model at large as well as infinite $U$, in the limit of infinite dimensions (Bethe lattice with infinite coordination).

Our approach here is two-fold. On the one hand, we have used the general structure of the Green's function and self-energy in the ECFL theory to obtain a useful analytical ansatz which reproduces quite well the rich and complex frequency-dependence of the DMFT selfenergy at not too low doping level. This ansatz relies on the lowest-order Fermi-liquid expansion of the two auxiliary ECFL self-energies $\Psi$ and $\chi$. Quite remarkably, the marked deviations from the Fermi-liquid form and the particle-hole asymmetry found in the physical single-particle self-energy can be accounted for by this underlying Fermi-liquid form of auxiliary quantities. In turn, the deviations observed between the DMFT results and this lowest-order ansatz at lower doping levels emphasize the need for corrections to FL behavior in $\Psi, \chi$ within the ECFL. This part of our study thus provides useful analytical insights into the DMFT description of the doping-driven Mott transition.

On the other hand, we have used the DMFT results (obtained here with a high-accuracy NRG solver) as a benchmark of the ECFL theory. Specifically, we have solved numerically the $O\left(\lambda^{2}\right)$ ECFL equations, appropriately simplified in the limit of large dimensions. For not too low doping levels, where this $O\left(\lambda^{2}\right)$ scheme is applicable, we found that the spectral properties agree well provided the comparison is made as a function of the scaled frequency $\omega / Z D$, with $Z$ the quasiparticle weight. A similar situation arises in comparing the ECFL method for the Anderson impurity model, where $Z$ is rapidly suppressed as the Kondo limit is approached ${ }^{43}$. This adjustment of the frequency scale compensates the known weakness of the $O\left(\lambda^{2}\right)$ theory in obtaining $Z$ quantitatively, and enables, to some extent, a preview of the results of the planned higher-order calculations in the ECFL projection parameter $\lambda$.

From a physics point of view, we now summarize the most significant insights provided by our study.

Doped Mott insulators are found to be characterized by a marked particle-hole dynamical asymmetry, as emphasized in recent ECFL ${ }^{44}$ and DMFT $^{9}$ studies. In the case of hole-doping, particle-like $(\omega>0)$ excitations are longer lived than hole-like $(\omega<0)$ ones, leading to more 'resilient' electron-like quasiparticles ${ }^{9}$. This dynamical asymmetry has physical implications for the spectral lineshapes ${ }^{9144}$ as well as thermopower ${ }^{9145}$. The asymmetric terms in the low-frequency expansion of the self-energy signal deviations from the Fermi-liquid theory which are usually ignored in weak-coupling studies. They become large at low hole doping and strong coupling, as demonstrated here in considerable detail, thus confirming the proposal made originally in Ref. 44 . 

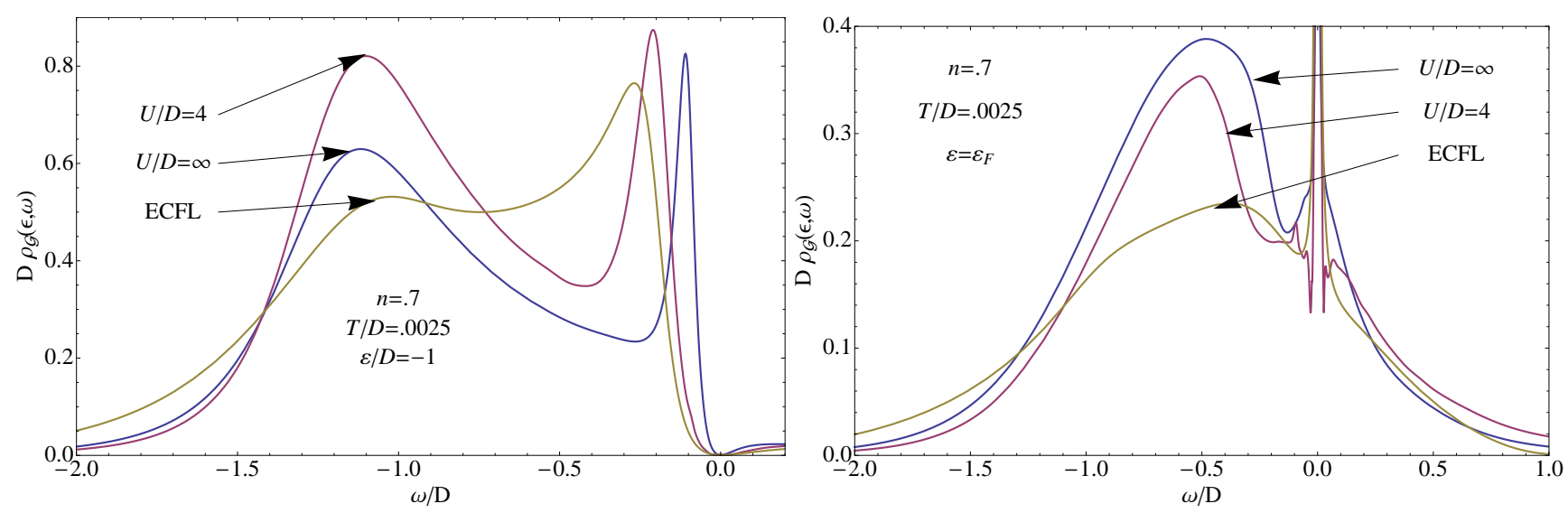

FIG. 12: (Color online) Spectral functions within the DMFT $(U=\infty$ and $U / D=4)$ and ECFL at two typical energies $\varepsilon=-D$ and $\varepsilon=\varepsilon_{F}$, with $n=0.7$ and $T=0.0025 D$. The location of the quasiparticle peak near $\omega \sim 0$ and the broad secondary peak for $\omega<0$ are common to both calculations. While there are subtle differences, especially in the magnitudes of the secondary peaks, the main features of the three calculations match at high and low frequency.

Due to the importance of this asymmetry, we found that the energy vs. momentum dispersion of the quasiparticle state quickly deviates on the $\omega>0$ side from its low-energy value (associated with the renormalized effective Fermi velocity). The deviation is towards a weaker dispersion, closer to the bare band value. This is a prediction of both ECFL and DMFT which could be tested experimentally once momentum-resolved spectroscopies are developed in order to address unoccupied states (the 'dark side' for photoemission).

Regarding ARPES lineshapes, we also emphasize that the recent successful comparison $\frac{617}{6}$ between the ECFL and the experimental ARPES lineshapes in the optimally doped and overdoped cuprates along the nodal direction can just as well be interpreted as the similar success of the DMFT interpretation of these lineshapes. The adjustment of the momentum dependence of the caparison factor for different systems in Refs. 6677hints at the importance of the momentum-dependence of the self-energy. This momentum-dependence is already present in the ECFL in two dimensions, and also emerges from cluster DMFT calculations.

Further comparison between the nature of the momentum dependence in both theories is to be addressed in future work. More generally, we believe that this work lays the foundation of a useful program where the momentumdependent self-energies can be reliably computed and expressed in simple analytic forms. While cluster DMFT methods can already provide some answers to this important problem, the ECFL theory readily treats low dimensions and the momentum dependence. In order to get further solid results, the current limitation of the ECFL to the somewhat overdoped regime needs to be overcome. This limitation arises from the low order of the expansion in $\lambda$, and brute-force higher order calculations in $\lambda$ are planned. In this task, the insights gained from the present comparison with DMFT, are invaluable.

\section{Acknowledgments}

A.G., J.M. and B.S.S. acknowledge useful discussions with Xiaoyu Deng at the initial stage of this project. The work at UCSC was supported by DOE-BES under Grant No. DE-FG02-06ER46319. A.G. and J.M. acknowledge the support of Ecole Polytechnique and Collège de France. R. Ž. and J. M. acknowledge the support of the Slovenian Research Agency (ARRS) under Program P10044.

\section{Appendix A: Quasiparticle occupation and $Z_{k}$ in the $O\left(\lambda^{2}\right)$ ECFL.}

The momentum distribution function, Fig. 15, shows good agreement with the DMFT at a density $n=.7$, and the large spillover for $k>k_{F}$ is of the same scale in both sets of calculation. Its importance in estimating the background spectrum in ARPES is well known, so this is already a reasonably reliable common result. It is also interesting that at the Fermi momentum, the magnitude of the distribution function is close to $\frac{1}{2}$ in both calculations, as argued in the literature ${ }^{15 / 46}$.

In Fig. 16 we compare the quasiparticle weight $Z$ in ECFL and DMFT. The $O\left(\lambda^{2}\right)$ ECFL result has some similarity to the Gutzwiller approximation $\frac{47}{4}$ result $2 \delta /(1+\delta)$ in the limited density range of validity. However it does not seem to vanish in any obvious way, if we extrapolate by eye to higher density $n$, highlighting its main weakness in the current state of development, but plotted against the effective density it becomes comparable to the $U / D=4$ DMFT curve over a limited range. 

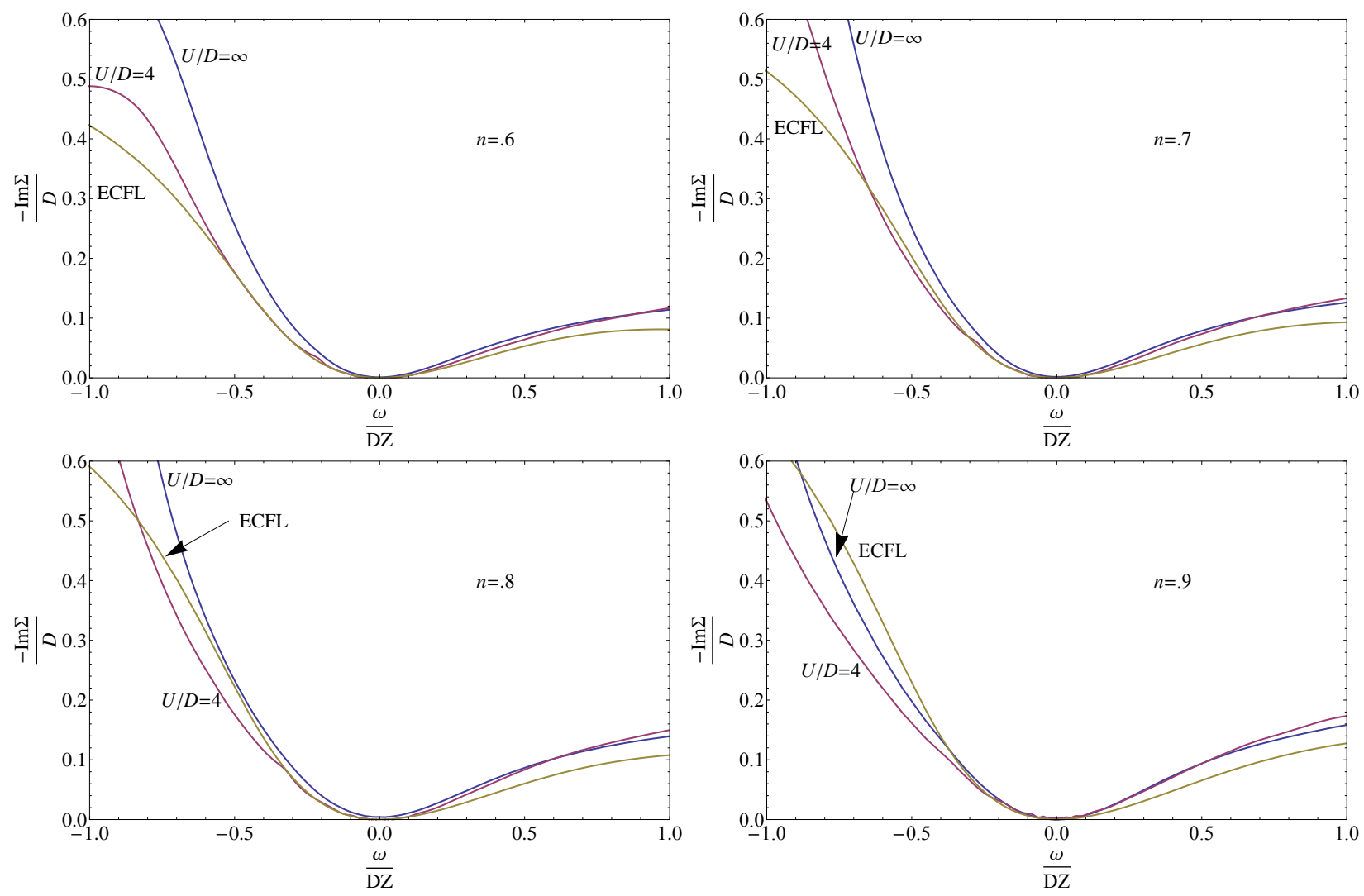

FIG. 13: (Color online) Spectral function (imaginary part) of the Dyson self-energy $\Sigma$ versus the scaled variable $\omega /(D Z)$ in the ECFL theory at order $\lambda^{2}$, and the DMFT at two values of $U$. The ECFL predicts a value of $\mathrm{Z}$ which is too large at low doping, and significant $U$ dependence creates differences between the $U / D=4$ and $U=\infty$ results of the DMFT. Nonetheless, all three cases overlap well at low frequencies when plotted against the scaled frequency. Surprisingly, this agreement survives to densities far beyond the expected range of the current version of the ECFL.

\section{Appendix B: NRG impurity solver convergence at small doping}

In order to obtain well-converged spectral functions using the NRG impurity solver at low doping $\delta$, several parameters in the method need to be apropriately tuned. Their choice affects both low-frequency and highfrequency parts of the spectral functions. In addition, it also significantly affects the numerical requirements both the duration of each NRG calculation and the number of the DMFT cycles until self-consistency. Very close to the Mott transition, obtaining fully converged results becomes computationally very expensive (several hundreds of DMFT cycles) even with Broyden acceleration 22 . In this section, we explore the effects of different choices on the quasiparticle residue $Z$ (low-frequency property), Fig. 17 and on the shape of the LHB (high-frequency property), Fig. 18.

We first explore the choice of the discretization scheme (i.e., how the coefficients of the Wilson chain are computed based from the input hybridization function). We compare the discretization scheme (denoted as Z) proposed by R. Žitko and Th. Pruschke in Ref. 22 which corrects the systematic discretization errors near band edges present both in the conventional discretization scheme (Y), Ref. 48, and in the improved scheme by V. Campo and L. Oliveira (C), Ref. 49. At low frequencies, one observes excellent overlap of the results, as indicated in Fig. $17 \mathrm{a}$. This is in line with the common wisdom that the NRG is a reliable method for low-frequency properties, having good spectral resolution in the vicinity of the Fermi level where the discretization grid is condensed. For this reason, the choice of the discretization scheme has little effect on $Z$. At high frequencies, however, one can clearly observe the systematic artifacts present in schemes $\mathrm{Y}$ and $\mathrm{C}$ : the LHB presents spurious (nonphysical) structure at the outer edge which is not present in the results of scheme Z, see Fig. 18 a. Recent comparisons of the NRG (using scheme Z) and continuoustime quantum Monte Carlo at finite temperatures have established that the NRG (using scheme Z) is, in fact, a rather reliable method also for high-frequency/finitetemperature properties. On the other hand, the NRG using schemes $\mathrm{Y}$ or $\mathrm{C}$ is expected to exhibit more pronounced systematic errors at high-frequencies and at finite temperatures.

The second important choice concerns the value of the discretization parameter $\Lambda$, which controls the coarseness 

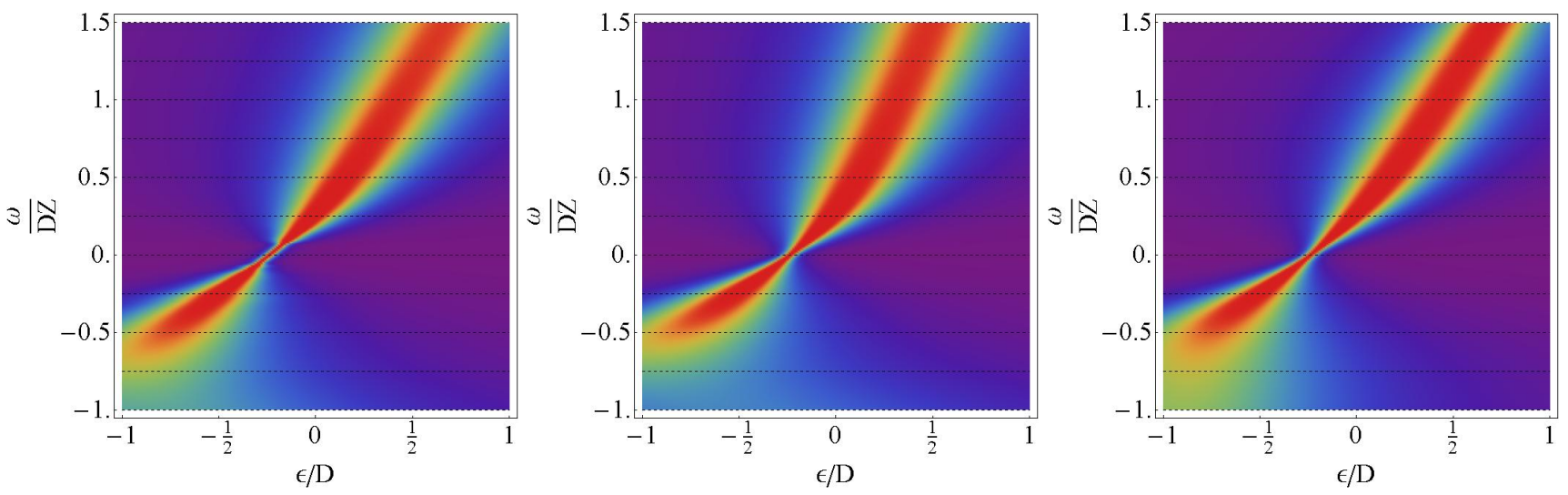

FIG. 14: (Color online) Physical spectral function $A(\epsilon, \omega)$. From left to right: $U=4$ DMFT, $U=\infty$ DMFT, and ECFL with $n=0.7$ and $T / D=0.0025$. Hot colors represent high intensity, while darker blue represents low intensity. Noting from the left panel of Fig. 12 that the QP band has a slightly different width in each calculation, we plot the spectral function here as a function of $\frac{\omega}{D Z}$. This brings the low energy (QP) features of the spectral function into impressive agreement, indicating that $\mathrm{Z}$, rather than $\delta$, is the fundamental energy scale of the extremely correlated state.
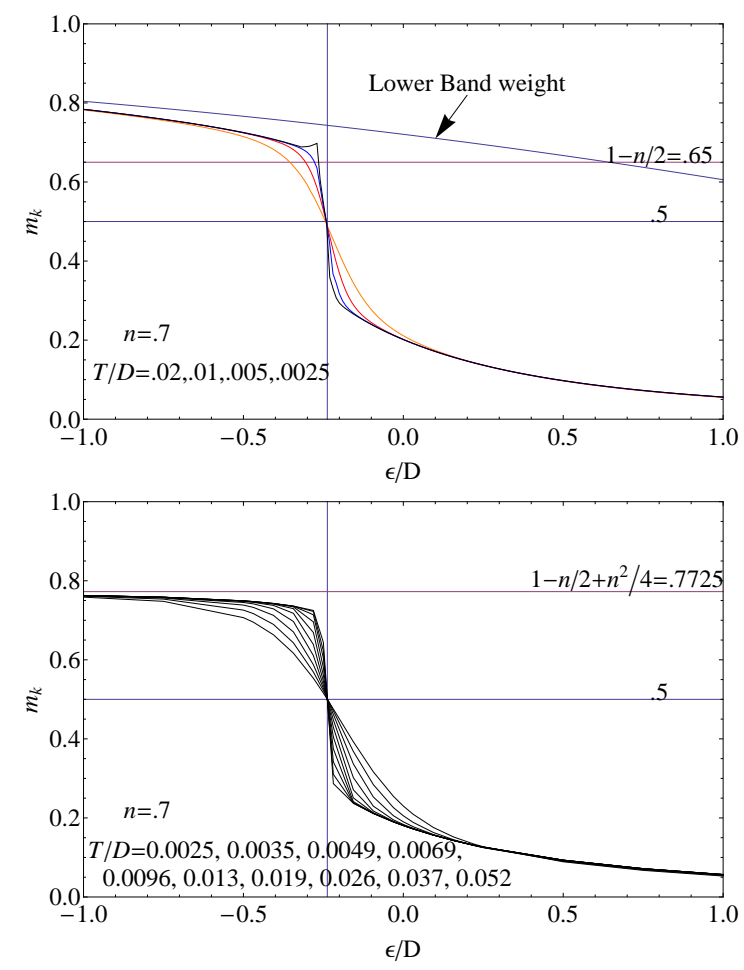

FIG. 15: (Color online) Momentum occupation versus $\varepsilon$ within the DMFT at $U / D=4$ (top) and the ECFL (bottom).

of the logarithmic grid. The standard choice is $\Lambda=2$, which is suitable to obtain well converged results at both low and high frequencies, see Figs. $17 \mathrm{p}$ and $18 \mathrm{~b}$. In fact, the results do not change much even when going to somewhat higher $\Lambda=2.5$, while for $\Lambda=3$ we start to observe some systematic deviations at very low doping $\delta$. We have also performed some test calculations for smaller values $\Lambda=1.9,1.8,1.7$; the results differ little from those

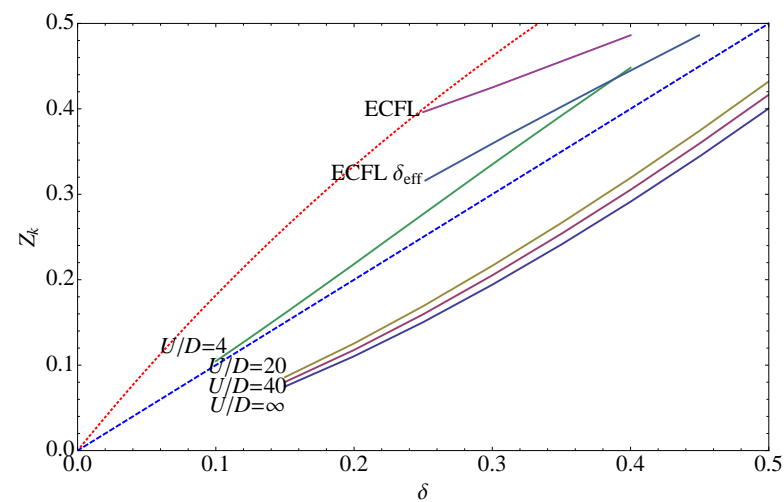

FIG. 16: (Color online) Quasiparticle weight $Z$ as a function of hole doping $\delta$ (or $\delta_{\text {eff }}=1-n_{\text {eff }}$, the effective hole doping) from the $O\left(\lambda^{2}\right)$ version of ECFL, and from DMFT for various values of U. More detailed DMFT results are in Fig. 1. The blue dashed line represents $Z=\delta$, the simplest $U=\infty$ slaveboson estimate, as a guide to the eye. The dotted red line represents the Gutzwiller approximation result $Z=\frac{2 \delta}{1+\delta}$.

for $\Lambda=2$ while being significantly more computationally expensive to produce.

We now consider the broadening parameter $\alpha$ which controls how the raw spectral function in the form of a set of weighted delta peaks is processed to obtain a smooth continuous representation. Too small values lead to spurious oscillations, too high values to overbroadening. These effects are nicely illustrated by the results for the LHB part of the spectral funciton in Fig. 18k. The long high-frequency tail of the LHB for increasing $\alpha$ is a clear over-broadening effect, while the oscillatory features for $\alpha=0.1$ are a discretization artifact. At low frequencies, the QP residue $Z$ converges as $\alpha$ is decreased, see Fig. 17k. We find that for $\alpha \leq 0.1$, the results practically overlap, while for $\alpha=0.2$ (the value used for most 

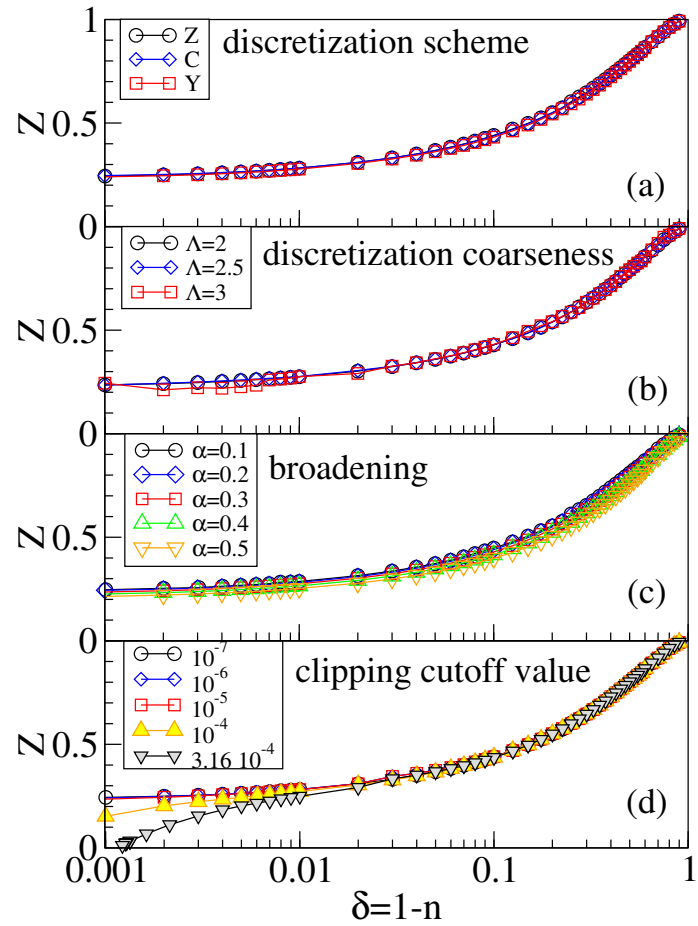

FIG. 17: (Color online) Quasiparticle residue $Z$ for the $U=$ $\infty$ Hubbard model as a function of doping $\delta$. We compare the DMFT results for different choices of the NRG impurity solver parameters. calculations in this work), the deviation from the asymptotic value is of order one percent. For large values of broadening (as commonly done in NRG calculations), $Z$ is underestimated. This is because the spectral weight is more spread around as $\alpha$ increases, thus less weight remains in the QP peak. Based on these results, we find that $\alpha=0.2$ is a good compromise.

Finally, we discuss a subtle issue which becomes important at very small dopings. The NRG discretization has difficulties if in the hybridization function there are extended regions of very low values. In particular, this leads to very slow approach to the self-consistency. For this reason, it is convenient to use a small, but finite cutoff value for the hybridization function to clip the input hybridization function to some minimum value at all frequencies. It is important, however, to choose this value so that the results are not perturbed. We find that using too high cut-off leads to incorrect $Z$ vs. $\delta$ behavior at low doping (a down-turn), see Fig. $17 \mathrm{~d}$. The effect is thus similar to overbroadening, since the spectral weight shifts from the quasiparticle peak to the region between the LHB and the QP peak, where the clipping is applied (for small $\delta$, where the LHB and the QP peak no longer overlap, but rather the $\mathrm{QP}$ becomes an isolated specgtral peak in the gap). There is also some effect of clipping on the LHB itself, Fig. 18d. Again, this effect is analogous to overbroadening.
1 A. Georges, G. Kotliar, W. Krauth, and M. J. Rozenberg, Reviews of Modern Physics 68, 13 (1996).

2 B. S. Shastry, Phys. Rev. Lett. 107, 056403 (2011), URL http://link.aps.org/doi/10.1103/PhysRevLett. 107.056403

3 B. S. Shastry, Phys. Rev. B 87, 125124 (2013), URL http: //link.aps.org/doi/10.1103/PhysRevB.87.125124

${ }^{4}$ B. S. Shastry, Phys. Rev. B 84, 165112 (2011), URL http: //link.aps.org/doi/10.1103/PhysRevB.84.165112,

5 E. Khatami, D. Hansen, E. Perepelitsky, M. Rigol, and B. S. Shastry, Phys. Rev. B 87, 161120 (2013), URL http: //link.aps.org/doi/10.1103/PhysRevB.87.161120.

${ }^{6}$ G.-H. Gweon, B. S. Shastry, and G. D. Gu, Phys. Rev. Lett. 107, 056404 (2011), URL http://link.aps.org/ doi/10.1103/PhysRevLett.107.056404.

7 Kazue Matsuyama and G.-H. Gweon, arXiv:1212.0299.

8 E. Perepelitsky and B. S. Shastry, "Extremely Correlated Fermi Liquids in the limit of infinite dimension", Annals of Physics (to appear) (2013), arXiv:.

9 X. Deng, J. Mravlje, R. Žitko, M. Ferrero, G. Kotliar, and A. Georges, Phys. Rev. Lett. 110, 086401 (2013), URL http://link.aps.org/doi/10.1103/PhysRevLett. 110.086401.

10 B. S. Shastry, Phys. Rev. Lett. 108, 029702 (2012), URL http://link.aps.org/doi/10.1103/PhysRevLett. 108.029702.

11 An early comment on Ref. 2] (K. Matho, Phys. Rev. Lett. 108, $029702(2012)$ ) and the response to this comment (B.S. Shastry, Phys. Rev. Lett. 108, 029702 (2012)) are noteworthy. In these works the momentum dependence of the Dyson self energy in the limit of large $d$ was discussed, before the exact simplifications arising from that limit were known precisely. As shown in Ref. 8 and in the present work, ECFL does provide a momentumindependent Dyson self energy in this limit.

12 A. Georges and G. Kotliar, Phys. Rev. B 45, 6479 (1992).

13 The minimal version of ECFL is obtained by setting to zero all correlation functions satisfying the Gutzwiller constraint- whereas the symmetrized version adds in some terms that are known to be zero, in order to make the effective Hamiltonian manifestly Hermitean ${ }^{3}$ (p 11).

14 To avoid the linear growth with $\omega$ of the self energy, one may define the Dyson Mori self energy, as in ${ }^{4}$ with a factor $1-\frac{n}{2}$ in the numerator of the Greens function. However in the present work, since DMFT directly yields the Dyson self energy, we simplify by quoting the results for this object directly.

15 D. Hansen and B. S. Shastry, Phys. Rev. B 87, 245101 (2013), URL http://link .aps .org/doi/10.1103/ PhysRevB.87.245101.

${ }^{16}$ A more complete discussion of the approach to the insulating limit within ECFL is not yet available. It is possible to also achieve a vanishing quasiparticle weight near halffilling in other ways as well, such as letting $c_{\chi}$ diverge as $\frac{1}{\delta}$. We prefer the current scheme for its simplicity and economy.

17 W. Metzner and D. Vollhardt, Phys. Rev. Lett. 62, 324 (1989). 


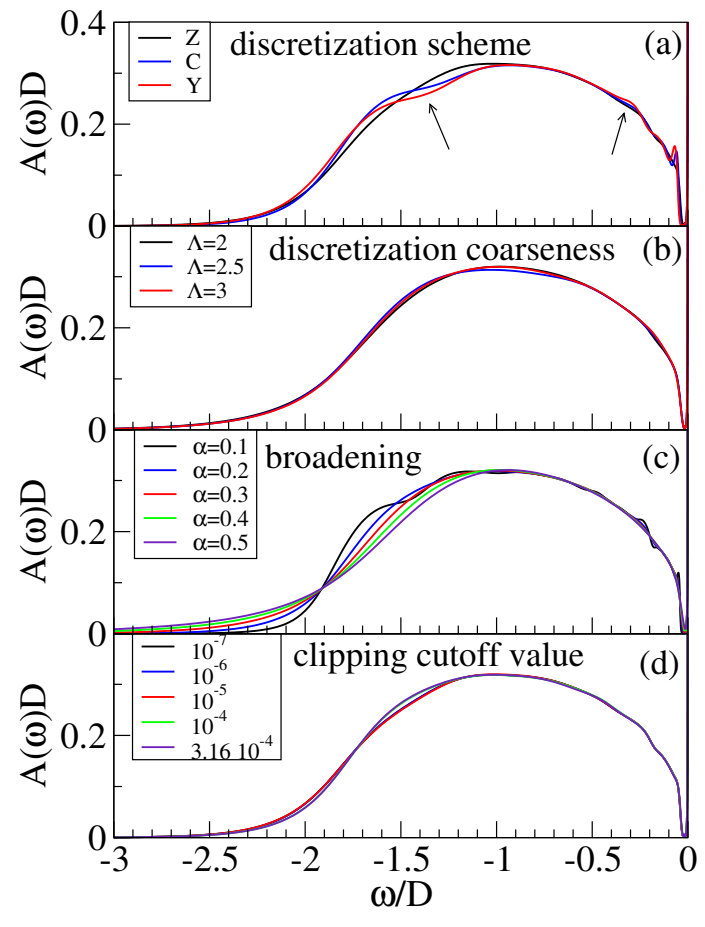

FIG. 18: (Color online) Lower Hubbard band part of the local spectral function $A(\omega)$ for the $U=\infty$ Hubbard model at low doping, $\delta=0.01$. In (a), the schemes $\mathrm{C}$ and $\mathrm{Y}$ produce spurious features at the outer band edge and at $\omega=0.3 D$ (indicated by arrows). In addition, the (expected) feature at the inner band edge at $\omega=0.1 D$ is overemphasized in $\mathrm{C}$ and Y schemes. In (d), we note that the curves for clipping values $10^{-7}, 10^{-6}$ and $10^{-5}$ nearly overlap, while those for $\geq 10^{-4}$ exhibit some deviations.

18 K. G. Wilson, Rev. Mod. Phys. 47, 773 (1975).

19 H. R. Krishna-murthy, J. W. Wilkins, and K. G. Wilson, Phys. Rev. B 21, 1003 (1980).

20 R. Bulla, Phys. Rev. Lett. 83, 136 (1999).

21 R. Bulla, T. Costi, and T. Pruschke, Rev. Mod. Phys. 80, 395 (2008).

22 R. Žitko and T. Pruschke, Phys. Rev. B 79, 085106 (2009).

23 R. Žitko, Phys. Rev. B 84, 085142 (2011).

24 A. Weichselbaum and J. von Delft, Phys. Rev. Lett. 99, 076402 (2007).

${ }^{25}$ R. Bulla, A. C. Hewson, and T. Pruschke, J. Phys.: Condens. Matter 10, 8365 (1998).

26 R. Peters, T. Pruschke, and F. B. Anders, Phys. Rev. B 74, 245114 (2006).

27 R. Žitko, Phys. Rev. B 80, 125125 (2009).

${ }^{28}$ W. F. Brinkman and T. M. Rice, Phys. Rev. B 2, 4302 (1970), URL http://link.aps.org/doi/10.1103/
PhysRevB.2.4302

${ }^{29}$ D. Vollhardt, Rev. Mod. Phys. 56, 99 (1984), URL http: //link.aps.org/doi/10.1103/RevModPhys.56.99

${ }^{30}$ P. Nozières, La méthode de Gutzwiller, Lecture Notes at Collège de France (1986), unpublished.

31 M. J. Rozenberg, G. Kotliar, and X. Y. Zhang, Phys. Rev. B 49, 10181 (1994), URL http://link.aps.org/doi/10. 1103/PhysRevB.49.10181.

32 S. Sakai, S. Blanc, M. Civelli, Y. Gallais, M. Cazayous, M.A. Measson, J. S. Wen, Z. J. Xu, G. D. Gu, G. Sangiovanni, et al., ArXiv e-prints (2012), 1207.5070.

33 G. Moeller, Q. Si, G. Kotliar, M. Rozenberg, and D. S. Fisher, Phys. Rev. Lett. 74, 2082 (1995), URL http:// link.aps.org/doi/10.1103/PhysRevLett.74.2082

${ }^{4}$ R. Bulla, T. Pruschke, and A. C. Hewson, Physica B Condensed Matter 259, 721 (1999).

35 M. Karski, C. Raas, and G. S. Uhrig, Phys. Rev. B 72, 113110 (2005).

36 M. Karski, C. Raas, and G. S. Uhrig, Phys. Rev. B 77, 075116 (2008).

37 Žiga Osolin and R. Žitko, Phys. Rev. B 87, 245135 (2013).

38 M. M. Zemljič, P. Prelovšek, and T. Tohyama, Phys. Rev. Lett. 100, 036402 (2008), URL http://link.aps.org/ doi/10.1103/PhysRevLett.100.036402

${ }^{3 y}$ K. Byczuk, M. Kollar, K. Held, Y.-F. Yang, I. A. Nekrasov, T. Pruschke, and D. Vollhardt, Nat Phys 3, 168 (2007), ISSN 1745-2473, URL http://dx.doi.org/ 10.1038/nphys538

${ }^{40}$ P. Grete, S. Schmitt, C. Raas, F. B. Anders, and G. S. Uhrig, Phys. Rev. B 84, 205104 (2011), URL http:// link.aps.org/doi/10.1103/PhysRevB.84.205104

${ }^{41}$ K. Held, R. Peters, and A. Toschi, Phys. Rev. Lett. 110, 246402 (2013).

42 M. Eckstein, M. Kollar, M. Potthoff, and D. Vollhardt, Physical Review B 75, 125103 (2007).

43 B. S. Shastry, E. Perepelitsky and A. C. Hewson, arXiv:1307.3492.

44 B. S. Shastry, Phys. Rev. Lett. 109, 067004 (2012), URL http://link.aps.org/doi/10.1103/PhysRevLett. 109.067004

45 K. Haule and G. Kotliar, in Properties and Applications of Thermoelectric Materials, edited by V. Zlatic and A. C. Hewson (Springer Netherlands, 2009), NATO Science for Peace and Security Series B: Physics and Biophysics, pp. 119-131.

46 R. R. P. Singh and R. L. Glenister, Phys. Rev. B 46, 14313 (1992), URL http://link.aps.org/doi/10.1103/ PhysRevB.46.14313

${ }^{4 r}$ T. M. Rice and K. Ueda, Phys. Rev. Lett. 55, 995 (1985).

48 M. Yoshida, M. A. Whitaker, and L. N. Oliveira, Phys. Rev. B 41, 9403 (1990).

49 V. L. Campo and L. N. Oliveira, Phys. Rev. B 72, 104432 (2005). 